\section{OPEN ACCESS}

Edited by:

Massimo Bonora,

Albert Einstein College of Medicine,

United States

Reviewed by:

Keith R. Laderoute,

SRI International, United States

Valeria Poli,

Università degli Studi di Torino, Italy

${ }^{*}$ Correspondence:

Margaret Ashcroft

m.ashcroft@medschl.cam.ac.uk

${ }^{\dagger}$ Present Address:

Thomas Briston

Hatfield Research Laboratories,

Neurology Innovation Centre, Eisai

Ltd., Hatfield, United Kingdom

‡These authors have contributed equally to this work

Specialty section:

This article was submitted to Molecular and Cellular Oncology,

a section of the journal

Frontiers in Oncology

Received: 06 April 2018

Accepted: 29 August 2018

Published: 04 October 2018

Citation:

Briston T, Stephen JM, Thomas LW, Esposito C, Chung Y-L, Syafruddin SE, Turmaine $M$ Maddalena LA, Greef B, Szabadkai G,

Maxwell PH, Vanharanta $S$ and

Ashcroft M (2018) VHL-Mediated

Regulation of $\mathrm{CHCHD} 4$ and Mitochondrial Function.

Front. Oncol. 8:388.

doi: 10.3389/fonc.2018.00388

\title{
VHL-Mediated Regulation of CHCHD4 and Mitochondrial Function
}

\section{Thomas Briston ${ }^{1+\neq}$, Jenna M. Stephen ${ }^{27}$, Luke W. Thomas², Cinzia Esposito ${ }^{2}$, Yuen-Li Chung ${ }^{3}$, Saiful E. Syafruddin ${ }^{4}$, Mark Turmaine ${ }^{5}$, Lucas A. Maddalena ${ }^{2}$, Basma Greef ${ }^{2}$, Gyorgy Szabadkai ${ }^{5,6,7}$, Patrick H. Maxwell ${ }^{8}$, Sakari Vanharanta ${ }^{4}$ and Margaret Ashcroft ${ }^{2 *}$}

\begin{abstract}
${ }^{1}$ Division of Medicine, Centre for Cell Signalling and Molecular Genetics, University College London, London, United Kingdom, ${ }^{2}$ Department of Medicine, University of Cambridge, Cambridge, United Kingdom, ${ }^{3}$ Cancer Research UK Cancer Imaging Centre, Institute of Cancer Research London, London, United Kingdom, ${ }^{4}$ Medical Research Council Cancer Unit, Hutchison/MRC Research Centre, University of Cambridge, Cambridge, United Kingdom, ${ }^{5}$ Division of Biosciences, Department of Cell and Developmental Biology, University College London, London, United Kingdom, ${ }^{6}$ The Francis Crick Institute, London, United Kingdom, ${ }^{7}$ Department of Biomedical Sciences, University of Padua, Padua, Italy, ${ }^{8}$ Cambridge Institute for Medical Research, University of Cambridge, Cambridge, United Kingdom
\end{abstract}

Dysregulated mitochondrial function is associated with the pathology of a wide range of diseases including renal disease and cancer. Thus, investigating regulators of mitochondrial function is of particular interest. Previous work has shown that the von Hippel-Lindau tumor suppressor protein ( $\mathrm{pVHL}$ ) regulates mitochondrial biogenesis and respiratory chain function. $\mathrm{pVHL}$ is best known as an E3-ubiquitin ligase for the $\alpha$-subunit of the hypoxia inducible factor (HIF) family of dimeric transcription factors. In normoxia, $\mathrm{pVHL}$ recognizes and binds hydroxylated $\mathrm{HIF}-\alpha(\mathrm{HIF}-1 \alpha$ and $\mathrm{HIF}-2 \alpha)$, targeting it for ubiquitination and proteasomal degradation. In this way, HIF transcriptional activity is tightly controlled at the level of HIF- $\alpha$ protein stability. At least $80 \%$ of clear cell renal carcinomas exhibit inactivation of the $\mathrm{VHL}$ gene, which leads to HIF- $\alpha$ protein stabilization and constitutive HIF activation. Constitutive HIF activation in renal carcinoma drives tumor progression and metastasis. Reconstitution of wild-type $\mathrm{VHL}$ protein (pVHL) in pVHL-defective renal carcinoma cells not only suppresses HIF activation and tumor growth, but also enhances mitochondrial respiratory chain function via mechanisms that are not fully elucidated. Here, we show that $\mathrm{pVHL}$ regulates mitochondrial function when re-expressed in $\mathrm{PVHL}$-defective 7860 and RCC10 renal carcinoma cells distinct from its regulation of HIF- $\alpha$. Expression of $\mathrm{CHCHD4}$, a key component of the disulphide relay system (DRS) involved in mitochondrial protein import within the intermembrane space (IMS) was elevated by pVHL re-expression alongside enhanced expression of respiratory chain subunits of complex I (NDUFB10) and complex IV (mtCO-2 and COX IV). These changes correlated with increased oxygen consumption rate (OCR) and dynamic changes in glucose and glutamine metabolism. Knockdown of HIF-2 $\alpha$ also led to increased OCR, and elevated expression of CHCHD4, NDUFB10, and COXIV in 7860 cells. Expression of pVHL mutant proteins (R200W, N78S, D126N, and S183L) that constitutively stabilize HIF- $\alpha$ but differentially promote glycolytic metabolism, were also 
found to differentially promote the pVHL-mediated mitochondrial phenotype. Parallel changes in mitochondrial morphology and the mitochondrial network were observed. Our study reveals a new role for $\mathrm{pVHL}$ in regulating $\mathrm{CHCHD} 4$ and mitochondrial function in renal carcinoma cells.

Keywords: von Hippel-Lindau protein (pVHL), hypoxia inducible factor, mitochondria, bioenergetics, metabolism, CHCHD4, respiratory chain

\section{INTRODUCTION}

Dysregulated mitochondrial function is associated with a broad range of diseases including renal disease (1) and cancer (2). Mitochondria are best known as the sites for cellular oxygen consumption, generating chemical energy in the form of adenosine triphosphate (ATP). In fact, mitochondria are not only central to controlling many cellular metabolic pathways, but form an intracellular network enabling them to move, interface with other organelles (nucleus, endoplasmic reticulum, lysosome) and signal to/from different cellular compartments and machinery $(3,4)$. Thus, mitochondria are involved in regulating a broad range of cellular activities.

Mitochondria communicate with the oxygen-sensing machinery and are involved in regulating the cellular response to hypoxia (5). The HIF (HIF-1 and HIF-2) dimeric transcription factors are central to mediating hypoxia responses in cells. Hypoxia and increased HIF activation occurs in most cancers, leading to the upregulation of a range of genes involved in diverse cellular processes including metabolism and cell survival $(6,7)$. HIF comprises a tightly regulated HIF- $\alpha$ subunit (HIF- $1 \alpha$ and HIF-2 $\alpha$ ) and constitutively expressed HIF-1 $\beta$ subunit (7). Under normal oxygen levels, HIF- $\alpha$ protein is continuously synthesized and degraded. Targeting of HIF- $\alpha$ protein for degradation is achieved through hydroxylation of conserved proline residues, catalyzed by the oxygen-sensing dioxygenases, prolyl hydroxylase domain (PHD) enzymes (7-9). Recognition of the hydroxylated residues in HIF- $\alpha$ subunits is under the control of the von Hippel-Lindau ( $\mathrm{pVHL}$ ) tumor suppressor protein (10-12). pVHL forms the substrate recognition and catalytic component of an E3-ligase complex which functions to polyubiquitinate HIF- $\alpha$ subunits, targeting them for degradation by the $26 \mathrm{~S}$ proteasome $(8,11,12)$.

Inactivation of $\mathrm{VHL}$ occurs in a large percentage of patients with clear cell renal cell carcinomas (the most common form of kidney cancer) (13). Loss of pVHL tumor suppressor function promotes unopposed HIF- $\alpha$ stabilization and constitutive HIF activation which is associated with tumor progression (14). Re-constitution of wild-type pVHL or patient-derived mutant $\mathrm{pVHL}$ proteins into pVHL-defective renal carcinoma cells has proved a useful approach for investigating pVHL function (15-19). Interestingly, re-expression of pVHL in renal carcinoma cells increases the expression and activity of certain respiratory chain subunits including complex IV (CIV) subunits, mtCO-2 and COX IV (also known as COX4I1, COX4-1, and COX IV-1) $[(18,19)$, Supplementary Table 1], increases oxygen consumption rate (OCR) and mitochondrial DNA (mtDNA) content $(20,21)$. Knockdown of HIF-1 $\alpha$ or HIF-2 $\alpha$ in pVHL-deficient renal carcinoma cells has been shown to enhance basal OCR, mtDNA content and increase COX IV protein levels $(20,21)$. Collectively, these previous studies have led to the idea that constitutive HIF activation in the context of pVHL-defective renal carcinoma cells negatively regulates mitochondrial function (20). However, increased expression of mitochondrial respiratory chain subunits observed upon pVHL re-expression in pVHL-defective renal carcinoma cells is not HIF- $\alpha$-dependent (21), suggesting that pVHL (positively) regulates mitochondrial function independently of its HIFregulatory role through molecular mechanisms that have yet to be fully elucidated.

Previously, we discovered that the coiled-coil helix coiled-coil helix $(\mathrm{CHCH})$ domain 4.1 (CHCHD4) mitochondrial import protein is crucial for regulating intracellular oxygenation, mitochondrial localization, and morphology (22, 23). CHCHD4 [also known as MIA40 (24)] provides an import and oxidoreductase-mediated protein folding function as a key component of the disulphide relay system (DRS) within the mitochondrial intermembrane space (IMS) (22-27). CHCHD4 substrates contain a twin- $\mathrm{CX}_{n} \mathrm{C}$ motif and include respiratory chain subunits of complex I (CI) and CIV (22, 28-30).

Here, we further explore the role of pVHL in regulating mitochondrial function, bioenergetics, and morphology. We investigate effects on CHCHD4, metabolism and the contribution of HIF- $2 \alpha$. We show that pVHL increases the expression of $\mathrm{CHCHD} 4$, respiratory chain subunits known to be CHCHD4 substrates $(28,29)$ and promotes changes in mitochondrial morphology when re-expressed in pVHLdefective renal carcinoma cells. Alongside, we show increased OCR and dynamic changes in glucose and glutamine utilization. Using a panel of pVHL mutants (R200W, N78S, S183L and $\mathrm{D} 126 \mathrm{~N})$ that are unable to degrade HIF- $\alpha$, but promote differential effects on glycolytic metabolism (31), we show that these mutants also differentially affected the pVHL-mediated mitochondrial phenotype. Collectively, our data provide new molecular insight into the role of pVHL in regulating mitochondrial function, bioenergetics and morphology in renal carcinoma cells.

\section{RESULTS}

\section{pVHL Re-expression Regulates Mitochondrial Protein Expression and Increases Basal OCR}

To explore the role of pVHL in the regulation of mitochondrial function, we used matched 7860 renal carcinoma cell lines stably 
expressing either an empty vector control (786O-EV) or reexpressing wild-type pVHL (786O-VHL) (15). $786 \mathrm{O}$ parental cells harbor a single nucleotide inactivating deletion in $V H L$, and as a result express constitutively stabilized HIF- $2 \alpha$ but do not exhibit detectable HIF-1 $\alpha$ protein (32) (Figure 1A). Consistent with Hervouet et al. (21), we found that pVHL re-expression enhanced the basal expression of respiratory chain subunits of both nuclear and mitochondrial genetic origin, including the CIV subunits mtCO-2 and COX IV (Figure 1A). The expression of a number of distinct nonelectron transporting mitochondrial proteins, voltage-dependent anion channel 1 (VDAC1), heat shock protein 60 (HSP60) and ATP5B were not changed by pVHL re-expression (Figure 1A). Evaluation of a panel of both nuclear (SDHA and COX4I1) and mitochondrial encoded (ND6, cytochrome $b$ and $m t C O$ 2) transcripts encoding mitochondrial proteins including those major regulators of mitochondrial translation showed no significant change (Figure 1B and Supplementary Figure 1), indicating the observed increase in mitochondrial respiratory subunit expression in the $786 \mathrm{O}-\mathrm{VHL}$ cells was not due to changes in mRNA.

$\mathrm{CHCHD} 4$ regulates the import of a range of mitochondrial proteins including respiratory chain subunits of CI and CIV (28-30). Bioinformatics analyses of the Cancer Genome Atlas (TCGA-KIRC) data collection showed significantly increased $\mathrm{CHCHD} 4$ expression in clear cell renal cell carcinoma (ccRCC) designated as VHL non-mutated compared with those designated as VHL mutated in the collection (Figure 1C). Interestingly, we found that pVHL re-expression enhanced $\mathrm{CHCHD} 4$ protein levels without significantly affecting CHCHD4 mRNA in $786 \mathrm{O}$ cells (Figures 1D,E). Furthermore, CHCHD4 knockdown reduced the levels of respiratory chains subunits of CI (NDUFB10), complex II (CII, SDHA), complex III (CII, UQCRC2), and CIV (COX IV) in 7860 cells (Figures 1F,G). Previously we have shown that elevated $\mathrm{CHCHD} 4$ expression significantly increases basal OCR and ATP levels in normoxia in tumor cells expressing functional pVHL (22). Notably, we found that prolonged exposure of $786 \mathrm{O}-\mathrm{EV}$ cells to hypoxia $\left(1 \% \mathrm{O}_{2}, 72 \mathrm{~h}\right)$ reduced the growth of $786 \mathrm{O}-$ VHL cells but had no significant effect on the growth of 786O-EV cells (Supplementary Figure 2B), indicating that pVHL re-expression may lead to an increased reliance on oxygen (and oxidative phosphorylation) for cell growth. In relation to this, and consistent previous studies $(20,21)$, we found that pVHL re-expression significantly increased basal OCR, maximal respiratory capacity (Figures 2A-C and Supplementary Figure 2) and ATP levels (Figure 2D). Collectively, these data indicate that pVHL affects the expression of CHCHD4 as well as the expression of other CHCHD4regulated respiratory chain subunits, basal OCR and ATP levels.

Knockdown of HIF- $\alpha$ in renal carcinoma cells has been shown to increase mitochondrial COX IV protein expression (21) and basal OCR (20), implying a role for constitutive HIF$\alpha$ in negatively regulating mitochondrial respiration (20). In agreement, we found that HIF-2 $\alpha$ knockdown (HIF-2 $\alpha$ KD) significantly enhanced basal OCR and maximal respiratory capacity in 7860 cells (Figure 2E). As expected, in parallel, we observed reduced expression of HIF- $2 \alpha$ protein and HIF $2 A$ mRNA levels, and a reduction in the levels of the HIF-2 target, GLUT-1 (Supplementary Figures 3A,B). Furthermore, we found that stable HIF-2 $\alpha$ knockdown led to elevated CHCHD4, NDUFB10, and COX IV protein levels (Figure 2F). These effects were more obvious with HIF-2 $\alpha$ shRNA(1) (Figures 2F,G) which gave better knockdown than HIF-2 $\alpha$ shRNA(2) (Supplementary Figure 3C). Alongside, we observed significantly reduced expression of HIF2A and HIF-2 target genes ( $V E G F a$ and CCND1) (Supplementary Figure 3D). These data suggest that constitutive HIF- $2 \alpha$ affects the expression of CHCHD4 and mitochondrial respiratory chain subunits NDUFB10 and COX IV in (pVHL-defective) renal carcinoma cells, and this is associated with HIF- $2 \alpha$-dependent regulation of OCR.

\section{pVHL Re-expression Promotes Dynamic Changes in Glucose and Glutamine Metabolism}

Previous studies have reported that re-expressing pVHL in RCC10 renal carcinoma cells results in changes in glycolytic metabolism (31, 33). Here, we performed metabolomics analysis of $786 \mathrm{O}-\mathrm{EV}$ and $786 \mathrm{O}-\mathrm{VHL}$ cells. Consistent with the observed pVHL-mediated increase in respiratory chain subunit expression and enhanced OCR (Figures 1, 2), we found a significant decrease in intracellular glucose levels alongside a corresponding significant increase in intracellular glutamine levels in 786O-VHL cells compared with 786O$\mathrm{EV}$ cells (Figures 3A,C). However, we found no significant change in glucose or glutamine uptake, which was measured in parallel by analyzing the media from $786 \mathrm{O}-\mathrm{VHL}$ and 786O-EV cells (Figures 3B,D). Collectively, these data indicate that $\mathrm{pVHL}$ re-expression leads to a change in glucose and glutamine utilization consistent with increased respiratory drive.

Culturing tumor cells in glucose-free (galactose-containing) media forces them to utilize the respiratory chain and produce ATP via oxidative phosphorylation (34). Thus to evaluate the contribution of HIF- $2 \alpha$ to respiration in the presence and absence of glucose, we measured basal OCR in $786 \mathrm{O}$ cells in the context of HIF-2 $\alpha$ knockdown (Figure 3E). As anticipated, shifting cells to glucose-free (galactose-containing) media significantly increased basal OCR (Figure 3E), and was observed for both control and HIF-2 $\alpha$ knockdown cells (Figure 3E). Notably, this effect on basal OCR was more significant for HIF- $2 \alpha$ knockdown cells than control cells (Figure 3E). Moreover, compared to control cells for each condition, HIF- $2 \alpha$ knockdown caused a similar increase in basal OCR in both glucose and glucose-free (galactose-containing) media, indicating that HIF-2 $\alpha$ loss in (pVHL defective) renal carcinoma cells can promote increased mitochondrial respiration under different metabolic environments. 


\section{A}

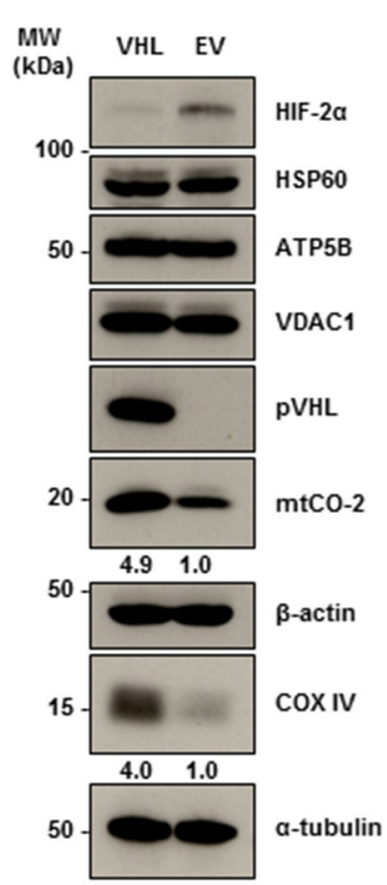

F

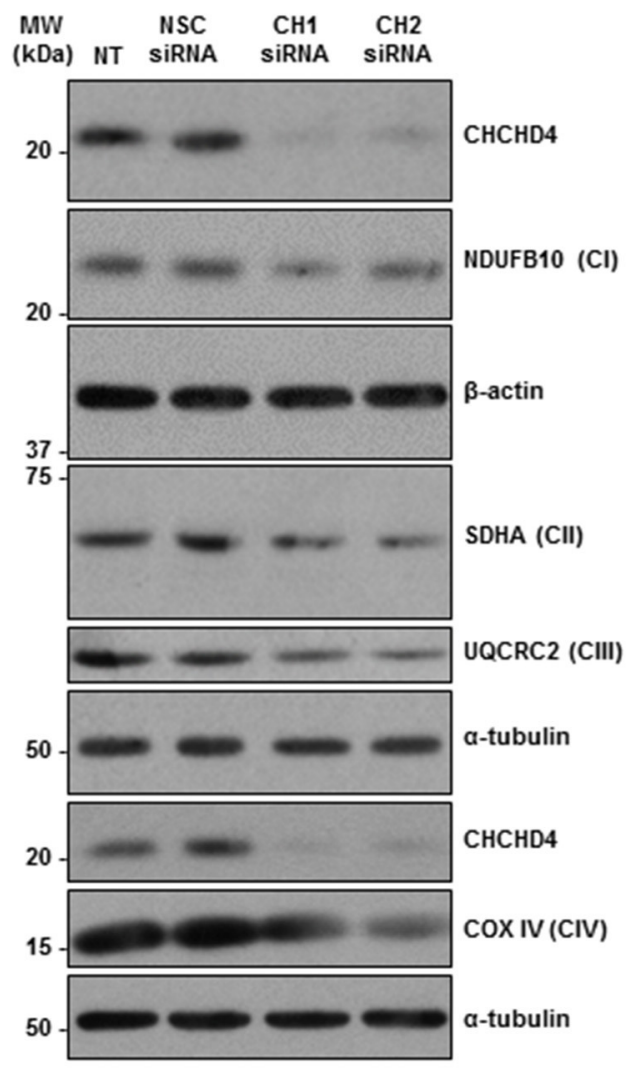

B

C
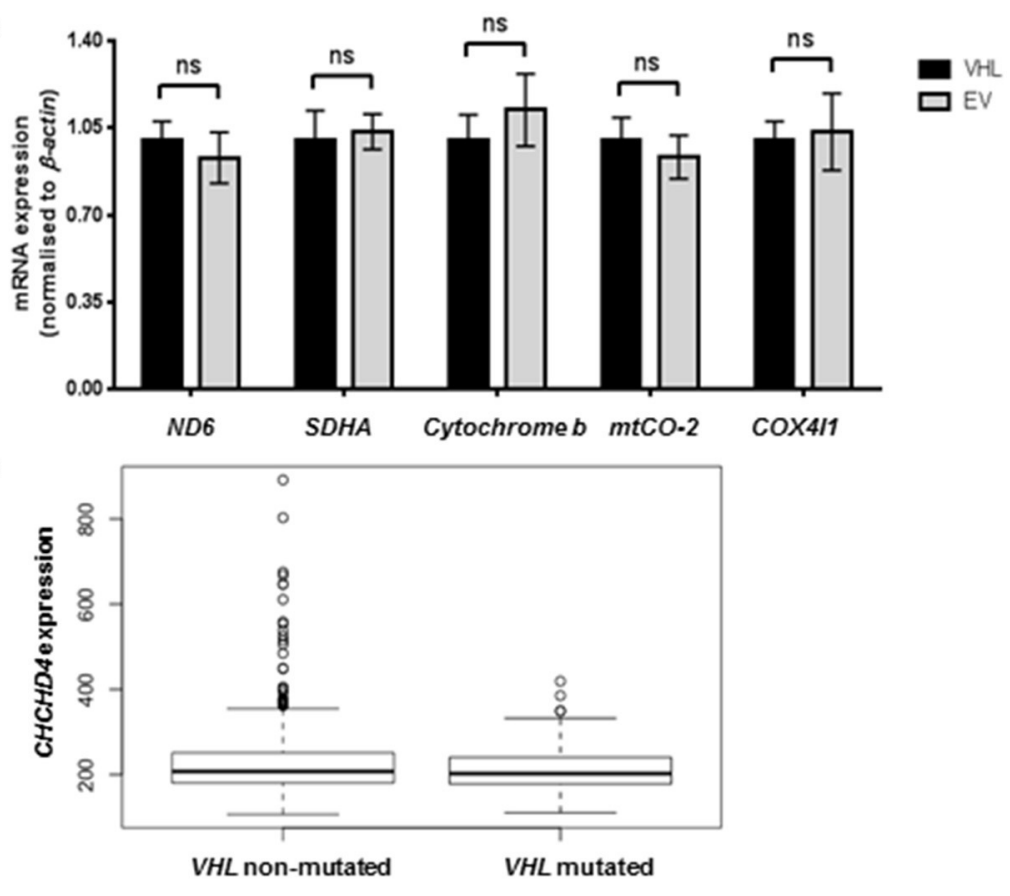

D

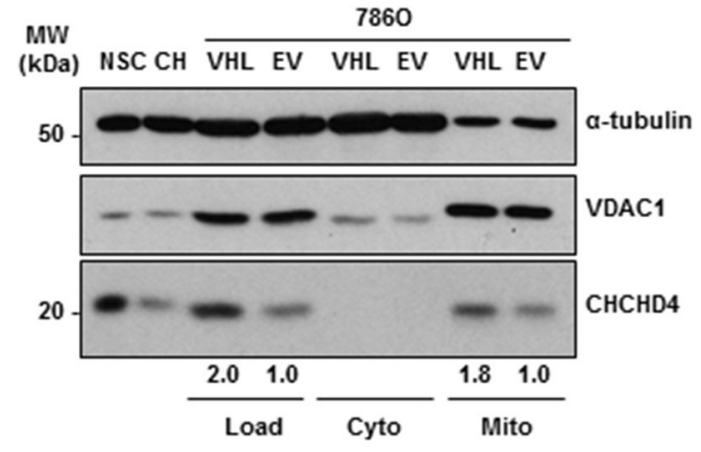

E

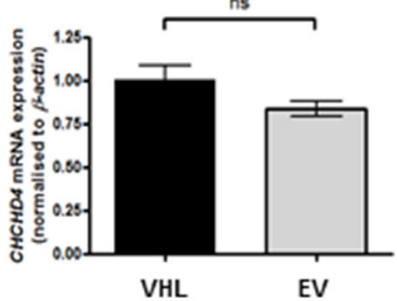

G

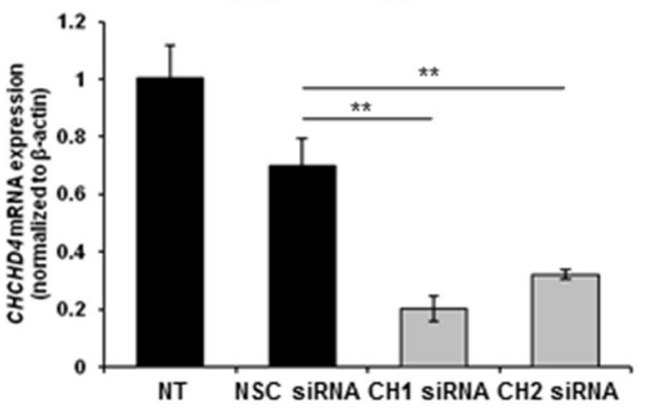

FIGURE 1 | pVHL re-expression regulates mitochondrial protein expression. (A) Western blot analysis of mitochondrial protein expression in 786O-VHL (VHL, stably re-expressing $\mathrm{PVHL}$ ) cells and 7860-EV (EV, vector control) cells. $\alpha$-tubulin and $\beta$-actin were used as load controls. Densitometric analysis of western blots show fold change in mtCO-2 and COXIV protein levels normalized to $\beta$-actin and $\alpha$-tubulin load controls respectively. (B) Graph shows relative expression of mRNA transcripts 
FIGURE 1 ( (ND6, SDHA, cytochrome b, mtCO-2, and COX M) in 7860-VHL (VHL) and 7860-EV (EV) cells, measured using RT-qPCR. Data were analyzed using the comparative Ct method $(n=4)$. (C) Boxplot of CHCHD4 expression in VHL mutated and VHL non-mutated ccRCC. TGCA-KIRC data was downloaded from cBioportal. $n=534$ patients were divided into VHL mutant $(n=202)$ and VHL non-mutant $(n=332)$ and analyzed for CHCHD4 expression in each group. Mean expression VHL non-mutated $=235.8, V H L$ mutated $=212.0$. Median values are shown for each group (black line), vertical size of the boxes are the interquartile range (IQR), and whiskers represent $1.5 \times$ IQR. Data were analyzed using a $t$-test with Welch's correction $p=0.00034$. (D) Western blots show CHCHD4 protein expression in total (Load), cytoplasmic (Cyto), and mitochondrial (Mito) fractions from 7860-VHL (VHL) and 7860-EV (EV) cells. HCT116 cells transiently expressing non-silencing control siRNA (NSC) or CHCHD4 siRNAs (CH), were used as controls for CHCHD4 protein expression (left two lanes). $\alpha$-tubulin and VDAC1 were used as cytoplasmic and mitochondrial protein markers respectively. (E) Graph shows relative expression of CHCHD4 mRNA expression in 7860-VHL and 7860-EV cells, measured using RT-qPCR. Data analyzed using the comparative Ct method. Data are presented as mean \pm S.E.M. $n=4$ (not significant (n.s.) $p>0.05)$. (F) Western blots show expression of mitochondrial proteins CHCHD4 and respiratory chain subunits NDUFB10 (CI), SDHA (CII), UQCRC2 (CIII), and COX IV (CIV) in 786O-EV cells transiently expressing non-silencing control siRNA (NSC, $20 \mathrm{nM}$ ) or two independent $\mathrm{CHCHD} 4$ siRNAs $(\mathrm{CH} 1$ and $\mathrm{CH} 2,20 \mathrm{nM})$. $\alpha$-tubulin and $\beta$-actin were used as a load controls. (G) Relative expression of $\mathrm{CHCHD4}$ mRNA in cells described in (A), measured using RT-qPCR. Data were analyzed using the comparative Ct method. Data are presented as mean \pm S.D. $n=3\left({ }^{\star \star} p<0.01\right)$.

\section{pVHL Mutants Differentially Regulate Mitochondrial Protein Expression, mtDNA Copy Number and ATP Levels}

To confirm the pVHL-dependent mitochondrial effects we observed in 7860 cells (Figures 1-3 and Supplementary Figures 1-3), we used RCC10 cells (35). RCC10 cells harbor an inactivating deletion in VHL (35), but in contrast to the $786 \mathrm{O}$ cells, RCC10 cells constitutively express both HIF- $1 \alpha$ and HIF- $2 \alpha$ proteins (Figure 4A). Pooled RCC10 cell lines independently stably expressing either control vector (pCMV), wild type pVHL (VHL), or a panel of mutant forms of pVHL proteins (R200W, N78S, D126N, and S183L) which are impaired in their ability to degrade HIF- $\alpha$ protein $(31,36)$ (Figure $\mathbf{4 A}$ and Supplementary Figure $\mathbf{4 A}$ ) were used. The R200W mutation in VHL is associated with congenital polycythemia (37), while its tumor suppressor activities are considered to be close to wild type VHL (38). The N78S, D126N, and S183L mutations in pVHL reduce its stability, and promote differential effects on glycolytic metabolism (31). As anticipated, these pVHL mutants were defective in blocking HIF activity and unable to rescue constitutive expression of HIF targets (GLUT1, BNIP3, and PHD3) (Figures 4A,B). However, whether these pVHL mutations differentially regulate mitochondrial protein expression and/or function is not known.

Consistent with our observations with 7860 cells (Figure 1), re-expression of pVHL in RCC10 cells led to an increase in $\mathrm{CHCHD} 4$, and respiratory chain subunits NDUFB10 (CI), SDHA (CII), UQCRC2 (CII), mtCO-2 (CIV), and COX IV (CIV) protein expression (Figure 4C and Supplementary Figures $4 \mathrm{~B}, \mathrm{C}$ ), while there was no change in other mitochondrial proteins, such as VDAC1 and prohibitin-1 (PHB1) (Supplementary Figures 4B,C). Intriguingly, expression of pVHL mutants had differential effects. Analysis of the R200W mutant revealed a similar phenotype to re-expression of $\mathrm{pVHL}$ (Figure 4C and Supplementary Figure 4). However, compared to the R200W mutant, the N78S mutant was less able to promote an increase in the panel of mitochondrial proteins evaluated (Figure 4C and Supplementary Figure 4), total cellular ATP (Figure 4D), or mtDNA copy number (Figure 4E). Expression of the N78S mutant in RCC10 cells has previously been shown to exhibit markedly reduced glucose utilization and lactate production compared to re-expression of wild type $\mathrm{PVHL}$, while two other mutants of pVHL, D126N and S183L were shown to have intermediate effects which were more similar to wild type pVHL (31). Like wild type pVHL, the D126N and S183L mutants also promoted a significant increase in mitochondrial proteins while the effect of the N78S mutant was less significant (Supplementary Figure 4). Together these data indicate the possibility that the pVHL-induced increase in mitochondrial proteins, ATP and mtDNA copy number is mechanistically separate from $\mathrm{pVHL}-$ mediated regulation HIF- $\alpha$.

\section{pVHL Mutants Differentially Regulate Mitochondrial Morphology and the Mitochondrial Network}

To further explore the relationship between the pVHL and mitochondria, 786O-VHL and 786O-EV cells were imaged using confocal and electron microscopy (EM) to assess mitochondrial morphology (Figures 5A,B). Clear differences in mitochondrial morphology between the 7860-VHL and 786O-EV cells were observed (Figures 5A,B). 786O-EV cells exhibited a more fused and elongated mitochondrial phenotype compared to $786 \mathrm{O}$ VHL which appeared to have smaller and more fragmented mitochondria (Figures 5A,B). Mitochondria appeared equally dispersed throughout the cell in both 786O-EV and 786O-VHL cells (Figure 5A). In agreement with the our images obtained by live-cell confocal imaging (Figure 5A), ultrastructural analysis by EM revealed that mitochondria appeared smaller and more rounded in $786 \mathrm{O}-\mathrm{VHL}$ cells, while mitochondria in $786 \mathrm{O}-\mathrm{EV}$ cells appeared more elongated, thinner and more tubular (Figure 5B). Quantification of mitochondrial length from the EM images showed the average mitochondrial length was significantly smaller in $786 \mathrm{O}-\mathrm{VHL}$ cells $(1.15 \mu \mathrm{m})$ compared to 7860-EV cells $(1.54 \mu \mathrm{m})$ (Figure 5C). Similar mitochondrial morphology changes were observed in RCC10 cells stably expressing vector control (pCMV) and wild type VHL (Figure 5D).

To further evaluate mitochondrial morphology, a panel of RCC10 cells stably expressing vector control (pCMV), wild type VHL (VHL), VHL mutants (R200W and N78S) described in Figure 5 were fixed, and the mitochondrial network examined using indirect immunofluorescence toward the ATP5B subunit of the $\mathrm{F}_{1} \mathrm{~F}_{\mathrm{O}}$-ATPase. ATP5B protein expression was unaffected by pVHL status (Figure 1A). Confocal imaging 


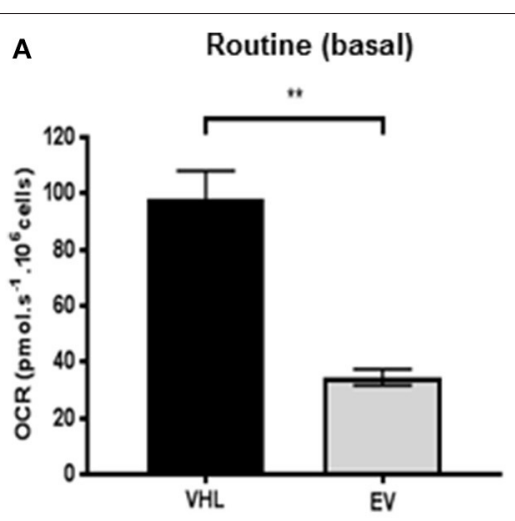

C

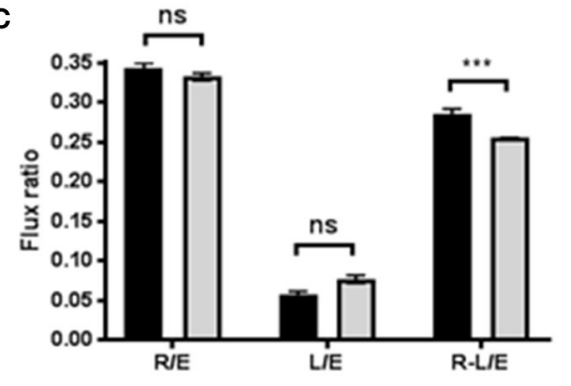

$\mathbf{F}$

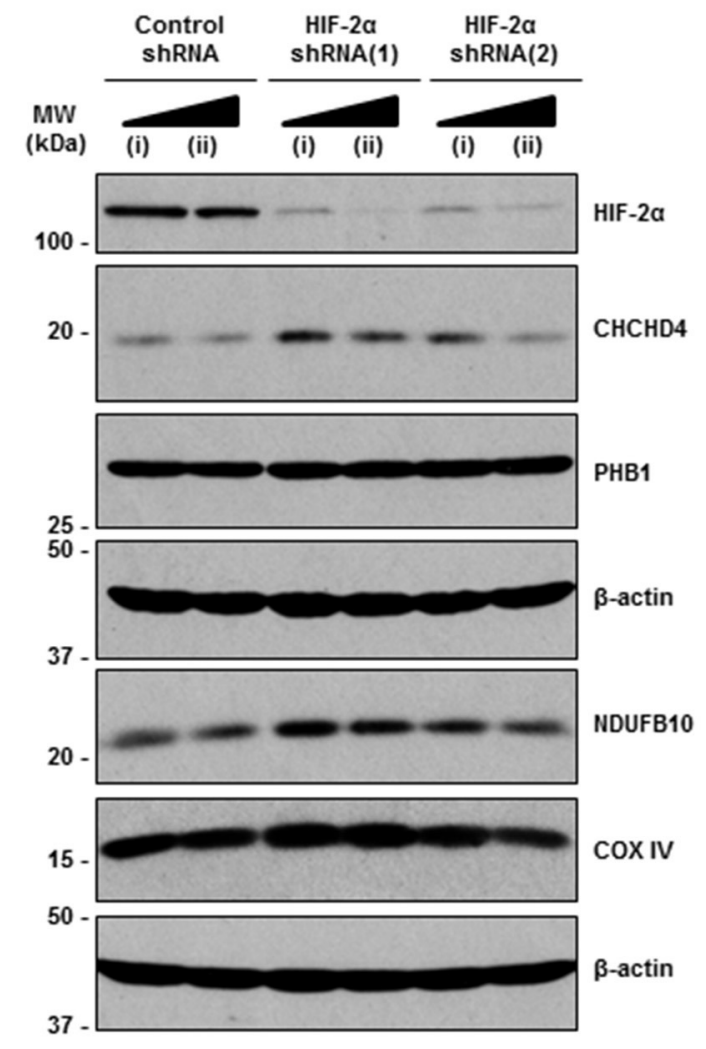

B

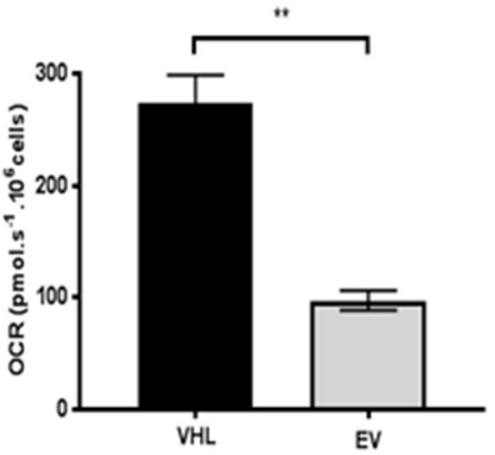

E
D

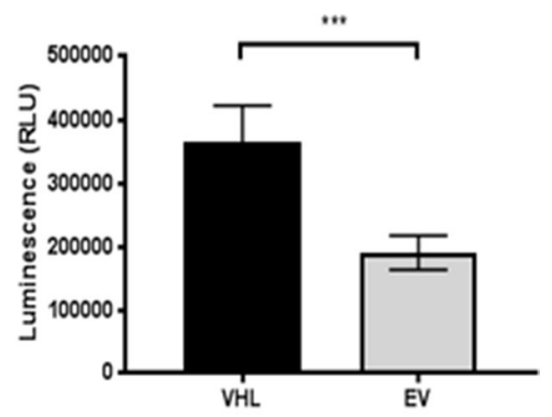

VHL

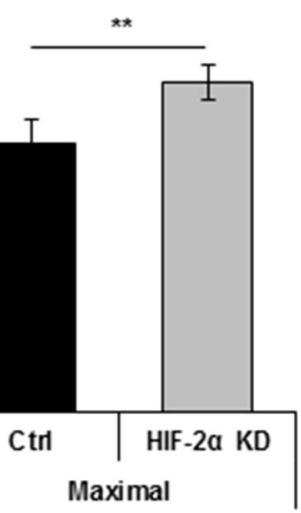

G
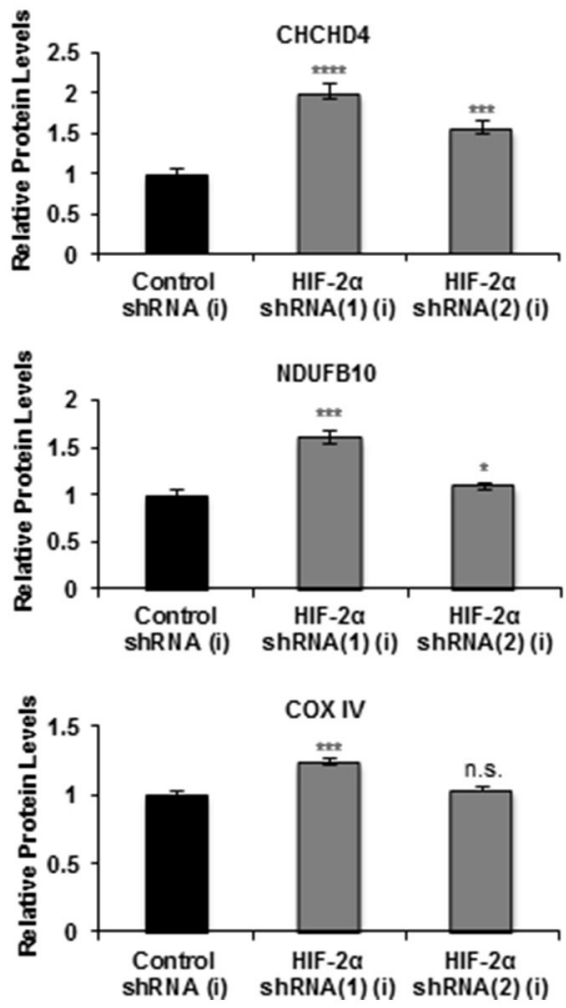

FIGURE 2 | pVHL re-expression or HIF-2 $\alpha$ knockdown increases basal OCR. (A,B) Graphs shows routine (basal) OCR (A) and maximal respiratory capacity (B) in 7860-VHL (VHL) and 7860-EV (EV) cells. OCR (pmol.s ${ }^{-1}$ ) was corrected for cell number (per $10^{6}$ cells) and non-mitochondrial respiration ( $\left.n=4\right)$. (C) Graph shows respiratory flux ratios (R/E, routine control ratio; L/E, leak control ration; R-L/E, net-routine control ratio) calculated from OCR data described in (A) $(n=4)$. (D) Graph 
FIGURE 2 | shows luminescence (RLU) as a measure of total cellular ATP content in 7860-VHL (VHL) and 786O-EV (EV) cells, normalized to cell number ( $n=3$ ). (E) Graph show routine (basal) OCR and maximal respiratory capacity in 7860-EV cells transiently expressing a non-silencing control siRNA (Ctrl) or HIF-2 $\alpha$ siRNA (HIF-2 $\alpha$ KD). Data are presented as mean \pm S.D. $n=4\left({ }^{*} p<0.05\right)$ and $\left({ }^{*} p<0.01\right)$. (F) Western blots show HIF-2 $\alpha$, COXIV and CHCHD4 protein levels in two independent 7860 cell pools (i and ii) stably transduced with increasing amounts of control shRNA and two independent HIF-2 $\alpha$ shRNAs(1) and (2). Data are representative of three independent experiments. (G) Graphs show relative levels of CHCHD4, NDUFB10 and COX IV protein from densitometric analysis of western data from experiments described in (F). Values were normalized to the load. Data are presented as mean \pm S.D. (n.s. $p>0.05,{ }^{*} p<0.05,{ }^{* * *} p<0.001$, and ${ }^{* * * *} p<0.0001$ ).

confirmed a similar change in mitochondrial morphology to $786 \mathrm{O}$ cells upon re-expression of pVHL (Figures 5A,D, 6A and Supplementary Figure 5A). Images were analyzed using the mitochondrial network analysis (MiNA) macro tool (39) (Figures 6B-E and Supplementary Figure 5A). RCC10 cells expressing the R200W mutant exhibited a similar mitochondrial morphology to cells re-expressing wild type pVHL (Figure 6), as they appeared fragmented and less tubular compared to the control (pCMV) cells (Figure 6A and Supplementary Figure 5A). We observed a larger mitochondrial footprint in wild type pVHL and R200W mutant cells compared to pCMV control cells as there was significantly more area covered by mitochondrial structures (Figure 6E). In contrast, RCC10 cells expressing the N78S mutant exhibited a mitochondrial morphology similar to the control (pCMV) cells (Figure 6), as they displayed a more elongated mitochondrial appearance compared to those re-expressing wild type pVHL (Figure 6). A similar trend in results were obtained using live cell imaging of the panel of RCC10 cells stained with the mitochondrial dyes MitoTracker red (Supplementary Figures 5B-E) and MitoView green (Supplementary Figure 5F), however the mitochondrial network analysis was not statistically significant (Supplementary Figure 5). Finally, consistent with a previous study (18), and other settings (40), we found no change in the expression of major regulators of mitochondrial biogenesis including PGC-1 $\alpha, P G C-1 \beta, N R F-1$, NRF-2 (Supplementary Figures 6A-D), while mtDNA copy number (Figure 4E and Supplementary Figure 6E) and TFAM expression (Supplementary Figures 6F,G) was increased upon pVHL re-expression. Taken together, these data indicate that pVHL regulated mitochondrial morphology correlates with changes in mitochondrial protein expression.

\section{DISCUSSION}

It is clear from previous studies that pVHL regulates mitochondrial function in renal carcinoma (see Supplementary Table 1). The negative regulation of the HIF- $\alpha$ subunits by pVHL has understandably been the focus of most attention and study, however other HIFindependent functions of pVHL exist (41). Here, we show that pVHL regulates mitochondrial protein expression, mtDNA copy number, respiration and mitochondrial morphology (Figure 6F).

Most strikingly, we found that expression of the mitochondrial DRS protein CHCHD4 $(22,23,29)$ was affected by pVHL status. CHCHD4 controls the import of a range of mitochondrial proteins into the IMS (28), and as an oxidoreductase and key component of the DRS, CHCHD4 along with ERV1/GFER introduces disulphide bonds into imported substrates (29). Previously, we have shown that elevated CHCHD4 expression increases basal OCR, controls intracellular oxygenation and regulates the mitochondrial network $(22,23)$. Here, pVHL reexpression led to increased levels of a range of respiratory chain subunits from CI-CIV including known CHCHD4 substrates [e.g., the CI subunit, NDUFB10 (30)]. Thus, pVHL affects CHCHD4 and mitochondrial function.

Reprogramming of glucose and glutamine metabolism occurs in renal carcinoma (42). Loss of pVHL function causes HIFmediated enhanced glycolysis and inhibition of mitochondrial function in renal carcinoma cells $(20,43)$. We found that knockdown of HIF-2 $\alpha$ in $786 \mathrm{O}$ cells caused elevated CHCHD4 and respiratory chain subunit expression and led to increased basal OCR, suggesting the possibility that HIF-dependent inhibition of mitochondrial function in pVHL mutated renal carcinoma involves CHCHD4. Small molecule inhibitors that target HIF show promise therapeutically in renal carcinoma $(17,44-46)$. Tumor cells rely on glycolysis and mitochondrial oxidative phosphorylation to survive (47). Thus, mitochondrial metabolism has become an increasingly attractive area for investigation and therapeutic exploitation in cancer (48). Our study suggests that blocking HIF-2 $\alpha$ in (pVHL-defective) renal carcinoma induces $\mathrm{CHCHD} 4$ and promotes a metabolic profile that could be exploited therapeutically. Thus, investigating the importance of $\mathrm{CHCHD} 4$ expression levels in renal carcinoma progression in the context of HIF dysregulation will be of particular interest. In fact our bioinformatics analysis using the Cancer Genome Atlas data collection (TCGA-KIRC) revealed that $\mathrm{CHCHD} 4$ expression was significantly lower in ccRCC patients designated with mutant VHL compared to those ccRCC patients designated with wild-type VHL (Figure 1C).

Along with changes in CHCHD4 and respiratory chain subunit expression, we found that pVHL re-expression caused a marked shift in fuel utilization: increased glucose and decreased glutamine utilization, consistent with the enhanced mitochondrial respiration. Using a panel of pVHL mutants N78S, D126N, and S183L that exhibit reduced stability and dysregulation of HIF- $\alpha$ but promote differential effects on glycolysis (31), we discovered that the pVHL-dependent effects on CHCHD4, mitochondrial protein expression and morphology directly correlated with pVHL-dependent regulation of metabolism. Our data suggest that pVHL can promote effects on CHCHD4 and mitochondrial function that are separable from its role in regulating HIF- $\alpha$. Our previous study suggests that CHCHD4 controls intracellular oxygenation and the 


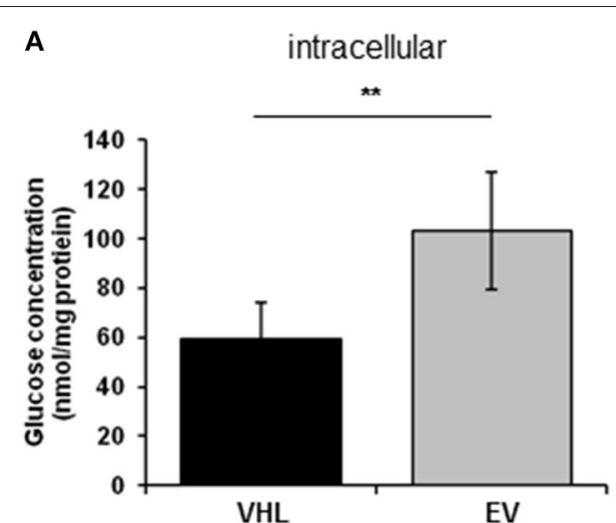

C

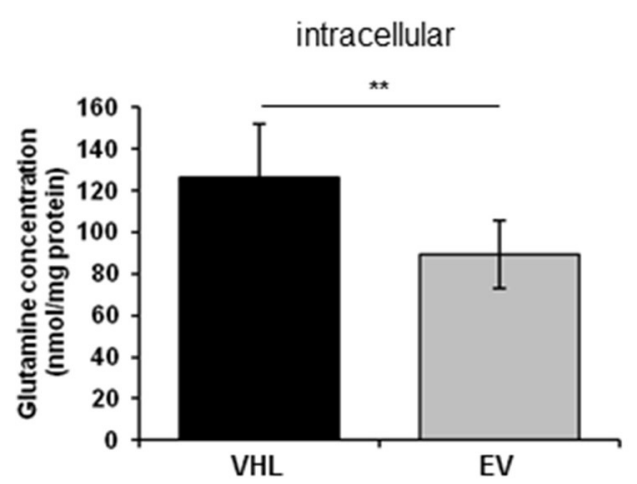

B

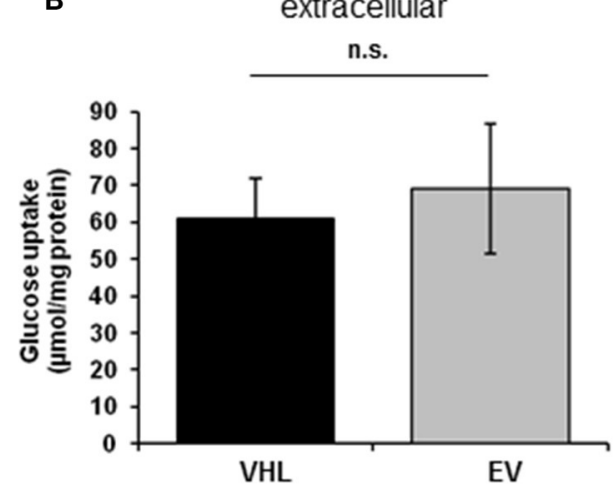

D

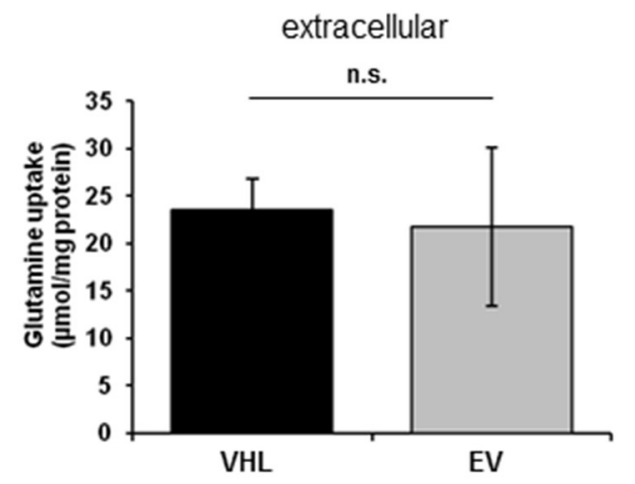

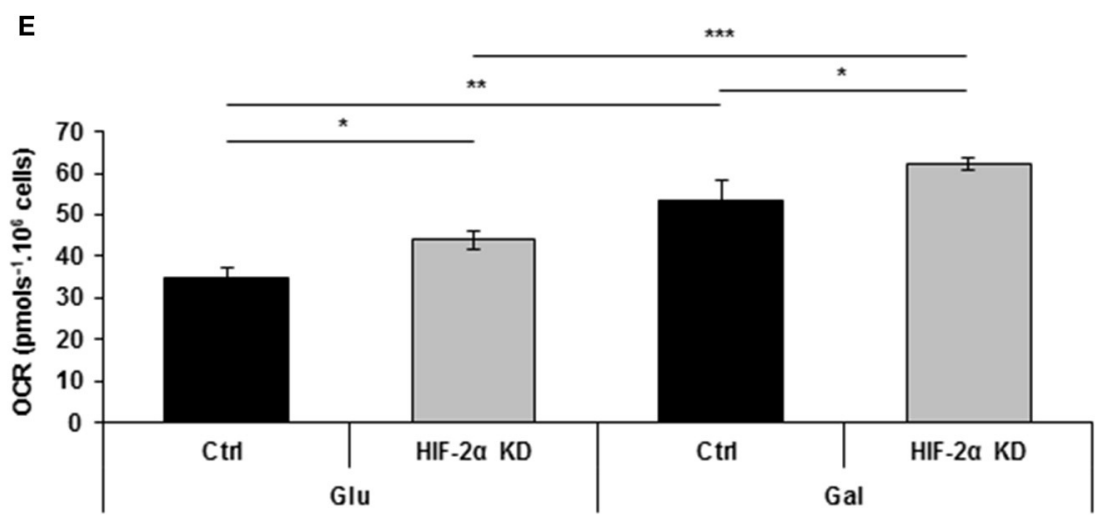

FIGURE 3 | pVHL re-expression promotes dynamic changes in glucose and glutamine metabolism. (A-D) Graphs show, (A) intracellular glucose levels ( $n=8)$, (B) glucose uptake from extracellular media $(n=8)$, (C) intracellular glutamine levels $(n=7)$, and (D) glutamine uptake from extracellular media $(n=7)$ in $786 \mathrm{O}-\mathrm{VHL}$ (VHL) and 7860-EV (EV) cells measured by magnetic resonance spectroscopy (MRS). $n=7-8$. (E) Graph shows basal OCR in 786O-EV cells transiently expressing a non-silencing control siRNA (Ctrl) or HIF-2 $\alpha$ siRNA (HIF-2 $\alpha \mathrm{KD}$ ) and cultured in glucose (Glu) or glucose-free (galactose, Gal) media. Data are presented as mean \pm S.D. (n.s. $p>0.05,{ }^{\star} p<0.05,{ }^{\star *} p<0.01$, and ${ }^{\star \star *} p<0.001$ ).

mitochondrial function upstream of HIF in the context of hypoxia (23). Future work determining the contribution of $\mathrm{CHCHD} 4$ and the DRS to pVHL-mediated regulation of mitochondrial protein expression, respiration, and mitochondrial morphology in the presence and absence of HIF- $\alpha$ will provide further mechanistic insight into the role of $\mathrm{CHCHD} 4$ in controlling mitochondrial function and metabolism. 

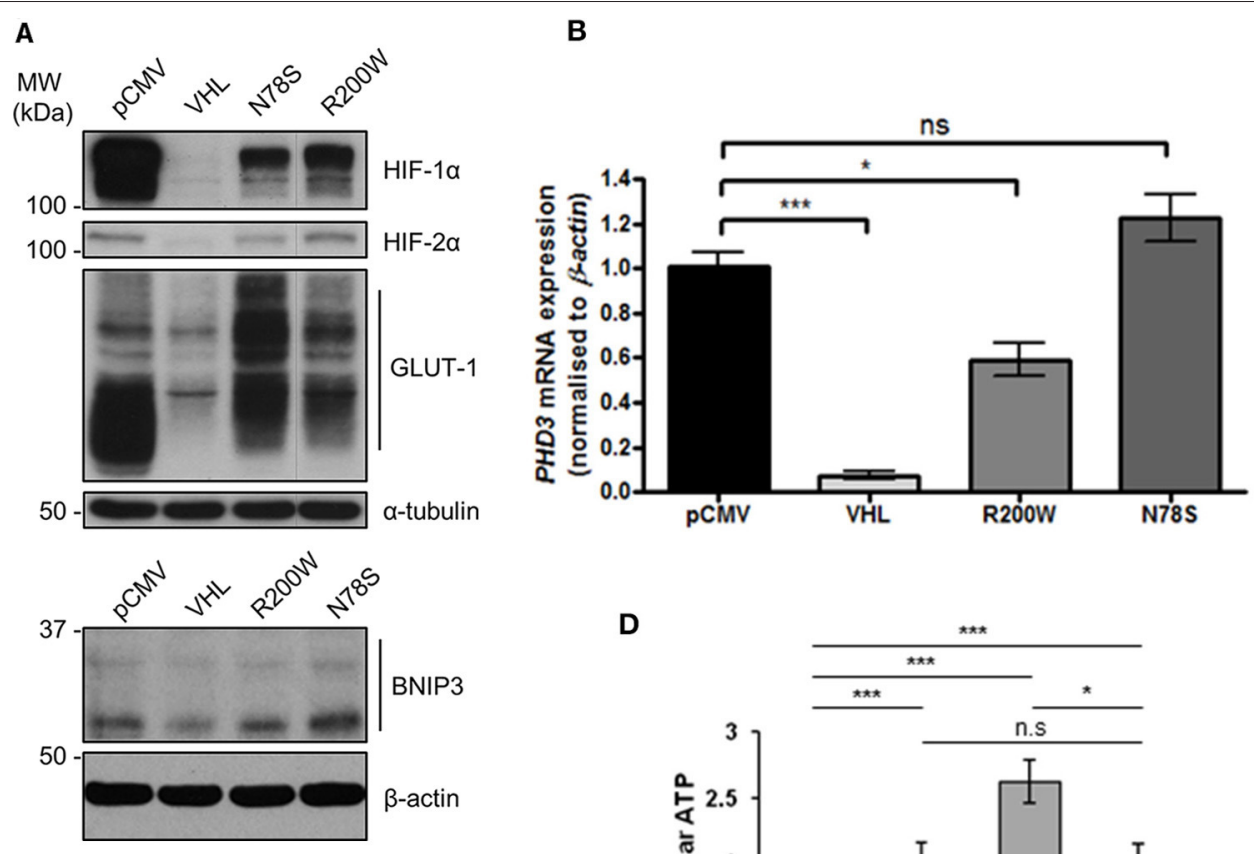

C

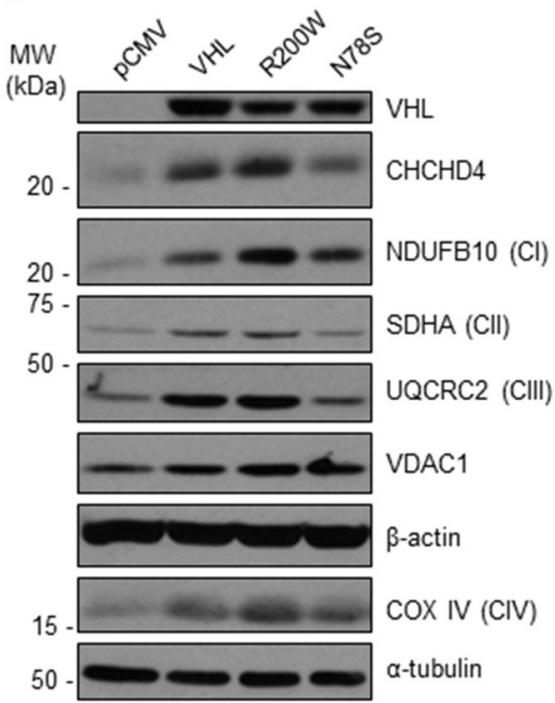

D

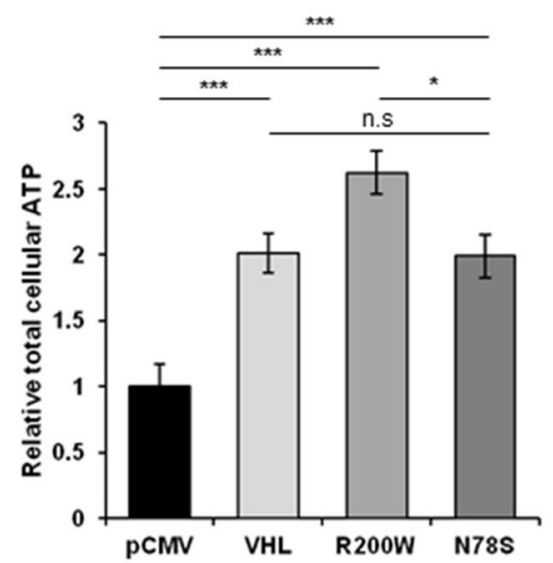

E

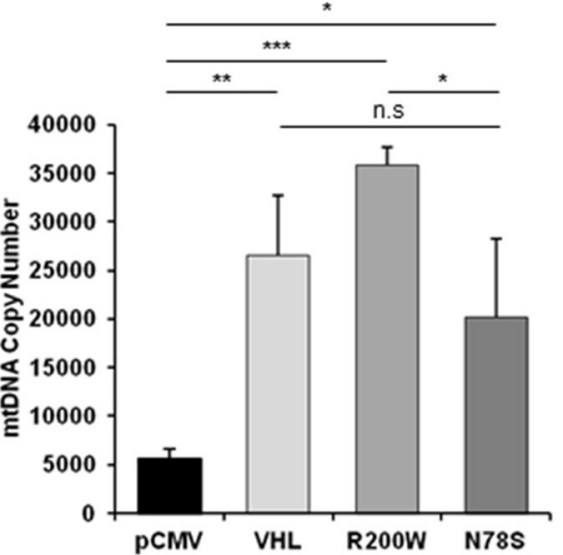

FIGURE 4 | pVHL mutants differentially regulate mitochondrial protein expression, mtDNA copy number and ATP levels. (A) Western blots show HIF-1 $\alpha, \mathrm{HIF}-2 \alpha$, GLUT-1, and BNIP3 protein levels in RCC10 cells expressing empty vector (pCMV), wild type pVHL (VHL), or pVHL mutants (R200W or N78S). $\alpha$-tubulin and $\beta$-actin were used as load controls. (B) Relative expression of PHD3 mRNA in RCC10 cells described in (A), measured using RT-qPCR. Data were analyzed using the comparative Ct method. Data are presented as mean \pm S.E.M. $n=3$ (n.s. $p>0.05,{ }^{*} p<0.05$, and ${ }^{* * *} p<0.001$ ). (C) Western blots show expression of mitochondrial proteins CHCHD4 and VDAC1, and respiratory chain subunits NDUFB10 (CI), SDHA (CII), UQCRC2 (CIII), COX IV (CIV) in RCC10 cells described in (A). pVHL expression was assessed as a control for re-expression, and $\beta$-actin and $\alpha$-tubulin were used as load controls. (D) Graph shows total cellular ATP content in RCC10 cells expressing wild type pVHL (VHL) or pVHL mutants (R200W or N78S), normalized to cell number $(n=4)$. (E) Graph shows mtDNA copy number in RCC10 cells expressing PVHL variants, calculated using the ratio of expression of mitochondrial ND1 gene to the single copy nuclear gene, $\beta 2 M$ to by RT-qPCR. Data in (D,E) are presented as mean \pm S.D. $n=6$ (n.s. $p>0.05,{ }^{*} p<0.05,{ }^{* *} p<0.01$, and ${ }^{* * *} p<0.001$ ). 
A
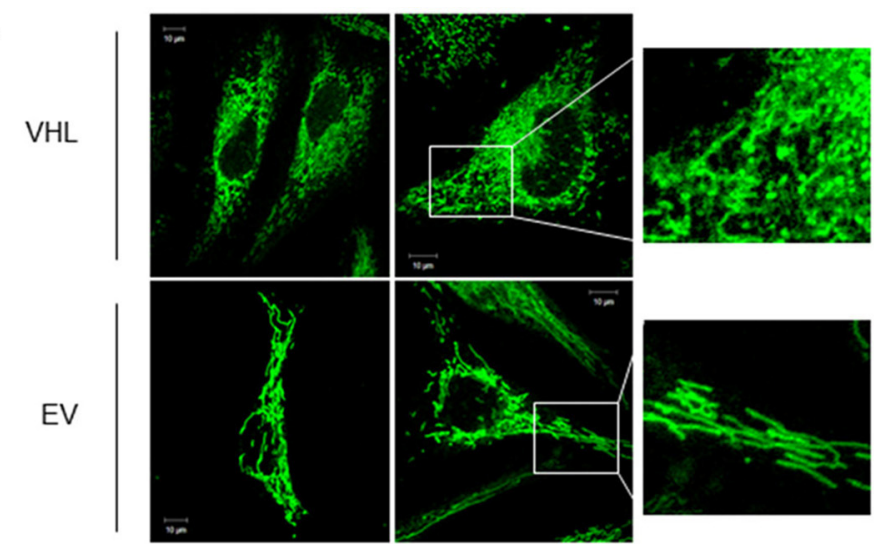

B

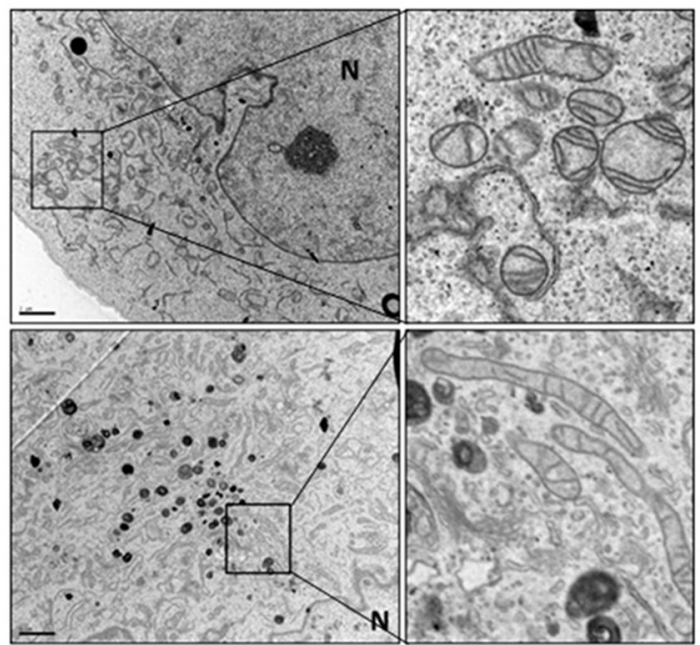

C

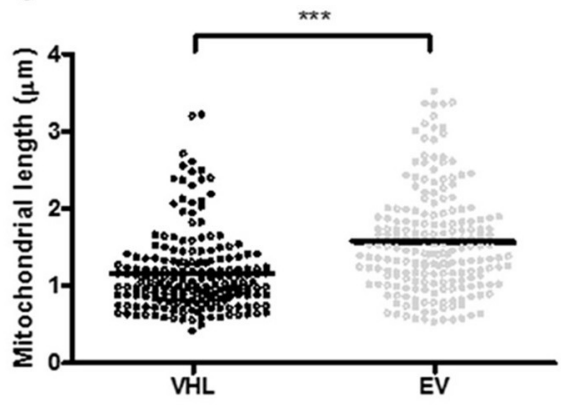

D
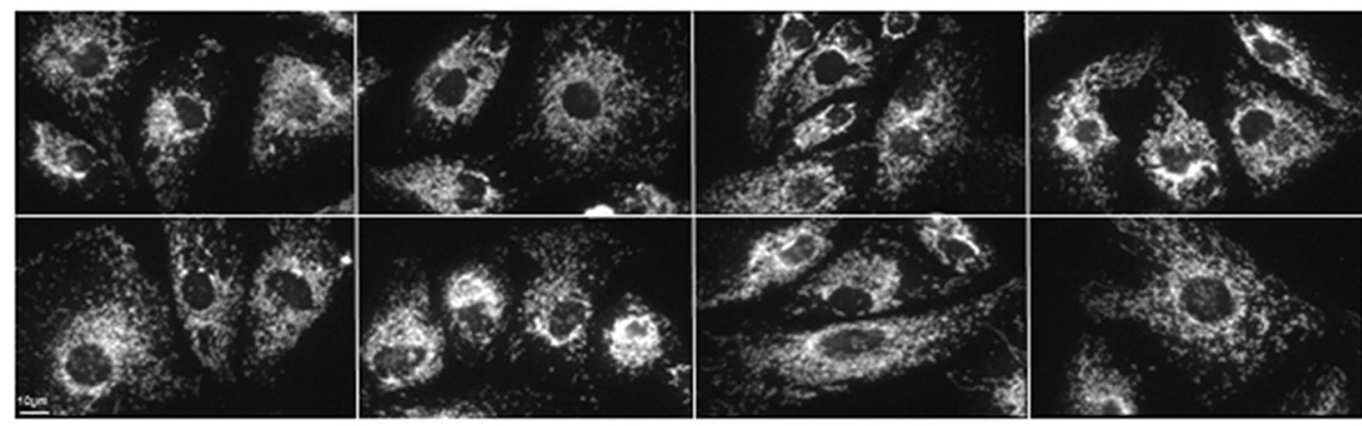

VHL
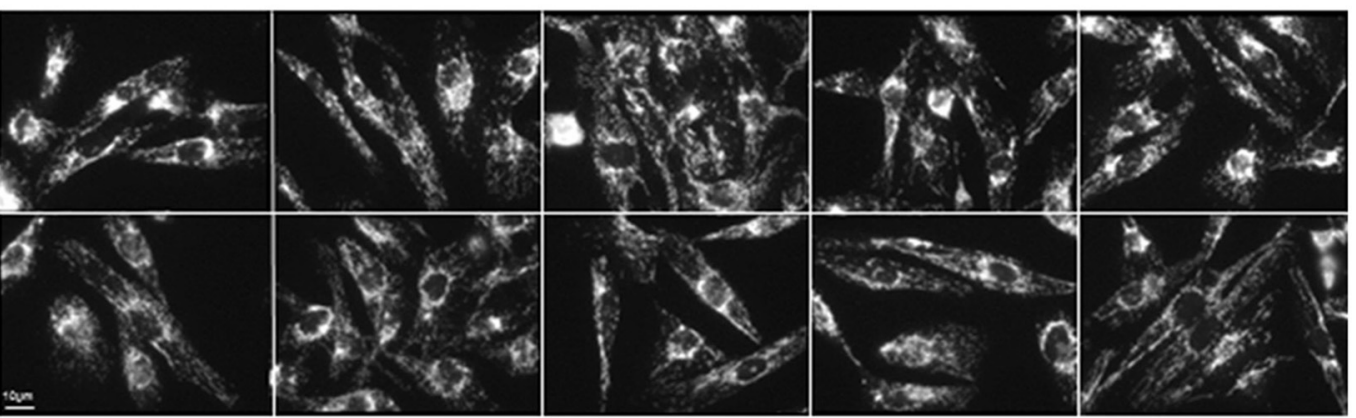

FIGURE 5 | pVHL re-expression affects mitochondrial morphology and the mitochondrial network. (A) Confocal microscope images of 786O-EV and 7860-VHL cells stained with MitoView Green (100 nM). Images were acquired with a Zeiss 510 META confocal laser scanning microscope using a $63 \times$ oil objective. Representative images and higher magnified images (right panel) are shown. (B) EM images of fixed 786O-VHL and 786O-EV cells. $\mathrm{N}=$ nucleus. Representative images and higher 
FIGURE 5 | magnified images (right panel) are shown. (C) Graph shows mitochondrial length measured from the EM images of fixed 786O-VHL (VHL) and 786O-EV (EV) cells described in (B). Mitochondrial length was measured from the most highly curved membrane through the central axis to the opposite periphery. Measurements were calculated using Image $\mathrm{J}$ software (National Institute of Health, Maryland) after calibration of scale using the scale bar. Over 200 mitochondria were scored from independent images of 10 cells $\left({ }^{\star \star \star} p<0.001\right)$. (D) Confocal microscope images of RCC10 cells empty vector (pCMV) and wild type pVHL (VHL) cells. Cells were fixed and mitochondria were stained for ATP5B. Images were acquired with a Zeiss 510 META confocal laser scanning microscope using a 63× oil objective.

\section{MATERIALS AND METHODS}

\section{Antibodies and Reagents}

All chemicals were purchased from Sigma Aldrich unless otherwise specified. The following antibodies were used for immunoblotting: anti-pVHL [BD Pharmagen (Ig32; 556347; 1:1,000)], anti- $\alpha$-tubulin (Sigma Aldrich; T6199; 1:5,000), antiCHCHD4 (Sigma Aldrich; HPA034688; 1:1,000), anti-HIF-2 $\alpha$ (Abcam; Ab199; 1:500), anti-ATP5B (Abcam; ab14730; 1:1,000), anti-mtCO-2 (Abcam; ab110258; 1:1,000), anti- $\beta$-actin (Abcam; 6276; 1:5,000), anti-HSP60 [Cell Signaling Technology (CST); \#4870; 1:1,000], anti-VDAC1 (Abcam; ab15895;:1,000), antiCOX IV (Abcam; ab14744; 1:1,000), anti-PHB1 (CST; \#2426; 1:1,000), anti-anti-GLUT1 (Alpha Diagnostics; GT12-A; 1:1,000), anti-DRP-1 (CST; \#5391S; 1:1,000), anti-pDRP-1 (Ser ${ }^{637}$ ) (CST; \#4867S; 1:1,000), anti-HIF-1 $\alpha$ (BD Biosciences; 610959; 1:1,000). Horseradish peroxidase (HRP)-conjugated secondary antibodies were purchased from Amersham and used at 1:5,000 dilution. For immunocytochemistry anti-ATP5B (Abcam; ab14730) was used at 1:500 dilution and Alexa Fluor 568 goat anti-mouse IgG (Invitrogen; A-11031) was used at 1:5,000 dilution.

\section{Cell Culture, siRNA Transfection, and shRNA Lentiviral Transduction}

The pVHL positive and negative 7860 cells were kindly gifted to us by William Kaelin Jr (Dana-Farber Cancer Institute, Harvard Medical School), and have been described previously (15). Stable pools of RCC10 cells expressing wild-type and mutant VHL were supplemented with $0.5 \mu \mathrm{M}$ G418 (Sigma Aldrich), and have been described previously (31). For shRNA lentivirus production, HEK293T cells were used. All cells were maintained in Dulbecco Modified Eagle Medium (DMEM; Invitrogen), supplemented with $10 \% \mathrm{FCS}$, L-glutamine $(2 \mathrm{mM})$, penicillin $(100 \mathrm{U} / \mathrm{ml})$ and streptomycin $(100 \mu \mathrm{g} / \mathrm{ml})$ at standard cell culture conditions $\left(37^{\circ} \mathrm{C}, 5 \% \mathrm{CO}_{2}\right.$ and $95 \%$ relative humidity). Transient transfection was performed using a nonsilencing control siRNA duplex (Qiagen; 5'-AATTCTCCGAA CGTGTCACGT- $\left.3^{\prime}\right)$ and custom made siRNA to human HIF$2 \alpha$ (Dharmacon; 5'-CCCGGATAGACTTATTGCCAA-3') (17, 49). Transient transfection with siRNA duplexes ( $20 \mathrm{nM})$ were performed using HiPerfect ${ }^{\mathrm{TM}}$ (Qiagen) or Lipofectamine 2000 (Invitrogen) transfection reagent according to manufacturer's instructions. Knockdown was confirmed by western blot analysis and real-time quantitative polymerase chain reaction (RT-qPCR) following $24 \mathrm{~h}$ siRNA incubation. For stable shRNA targeting of $H I F-2 A$, we used the shRNA expression vector, sGEP [a gift from J. Zuber, IMP, Austria (50)]. The two independent HIF-2A shRNA oligonucleotide sequences used for cloning are listed in Supplementary Table 3, and were obtained from Sigma
Aldrich. For shRNA lentivirus production, HEK293T cells were transfected with a mixture of the HIF-2A shRNA sGEP vector, psPAX2 and pMD2.G using FuGENE 6 transfection reagent (Promega E269A). The media containing the lentivirus was collected $72 \mathrm{~h}$ post-transfection and filtered through a $0.45 \mu \mathrm{M}$ PVDG sterile filter. 7860 cells obtained from J. Massague (MSKCC, New York) were transduced with the lentiviral supernatant in the presence of $8 \mu \mathrm{g} / \mathrm{mL}$ polybrene (Milipore) and selected with $4 \mu \mathrm{g} / \mathrm{mL}$ puromycin (Invivogen) for $48 \mathrm{~h}$ post-transduction. Stable selected cell pools were expanded and analyzed.

\section{RNA Isolation and Complementary DNA Synthesis}

Total RNA was extracted from cells using RNeasy mini kit with DNase digestion (Qiagen) as manufacturer's instructions. One microgram of total RNA was used for first strand synthesis using the qScript First-Strand cDNA Synthesis System (Quanta Biosciences). Complementary DNA (cDNA) was synthesized using the G-Storm thermocycler as per manufacturer's instructions.

\section{Real-Time Quantitative Polymerase Chain Reaction (RT-qPCR)}

Forward and reverse primers were used at a final concentration of $0.5 \mu \mathrm{M}$ and Mesa Blue SYBR green mix (Eurogentec) was used as per manufacturer instruction. Data was analyzed using the comparative cycle threshold (Ct) method using $\beta$-actin as the internal control. Values from technical replicates were averaged and analyzed using the equation below and as published (51):

Relative expression $\left(2^{-\Delta \Delta \mathrm{Ct}}\right)=[(\mathrm{Ct}$ gene of interest $-\mathrm{Ct}$ internal control) sample A - (Ct gene of interest - Ct internal control) sample B].

PCR primers were obtained from Sigma Aldrich and sequences are outlined in Supplementary Table 2.

\section{Mitochondrial Fractionation}

Mitochondria were isolated using differential centrifugation. Briefly, cells from confluent $15 \mathrm{~cm}$ plates were trypsinised and washed in PBS. Cells were re-suspended in homogenization buffer (250 mM mannitol, $0.5 \mathrm{mM}$ 2-[4-(2hydroxyethyl)piperazin-1-yl]ethanesulfonic acid (HEPES) $\mathrm{pH} 7.4$ and $0.5 \mathrm{mM}$ ethylene glycol-bis(2-aminoethylether)$N, N, N^{\prime}, N^{\prime}$-tetraacetic acid (EGTA), 100x EDTA-free protease inhibitor cocktail). Cells were homogenized with a glass-on-glass Dounce homogenizer using 100 strokes of a tight fitting pestle. A small volume of the total cell homogenate was removed and stored (load fraction). The homogenate was centrifuged at 900 $g$ for $10 \mathrm{~min}$ to pellet nuclei and intact cells, the supernatant 
A

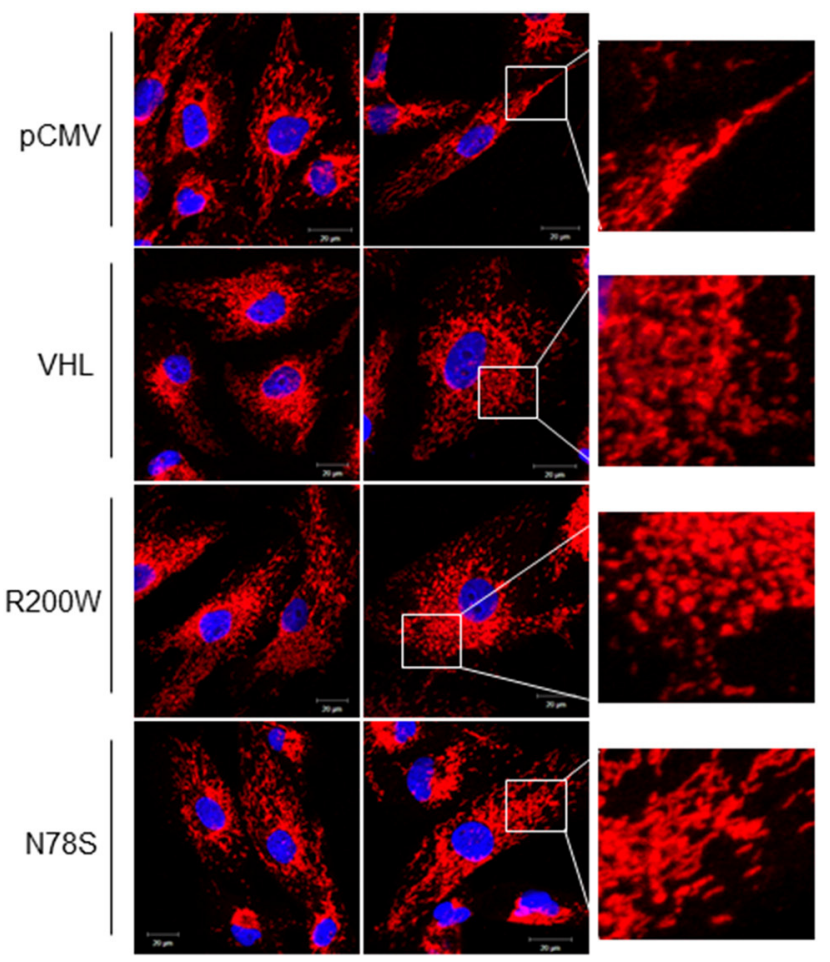

B

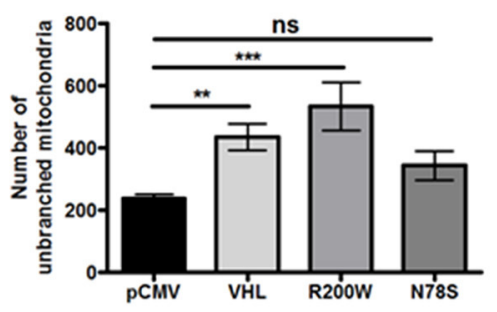

D

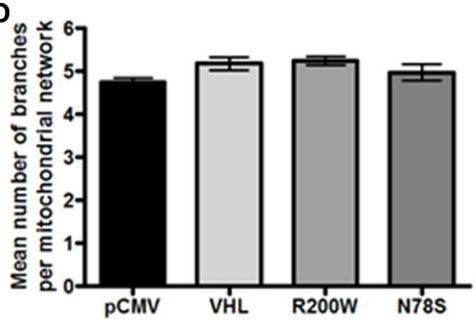

F
C

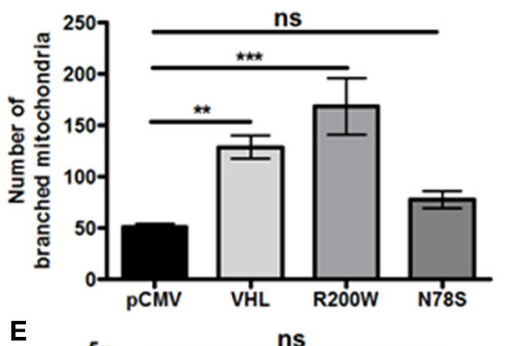

E

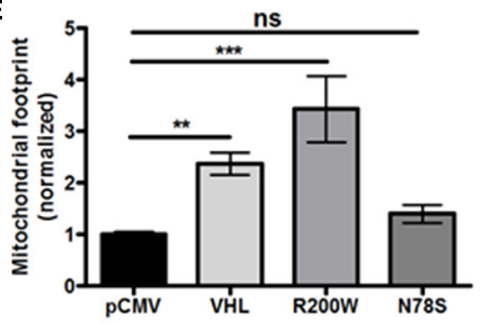

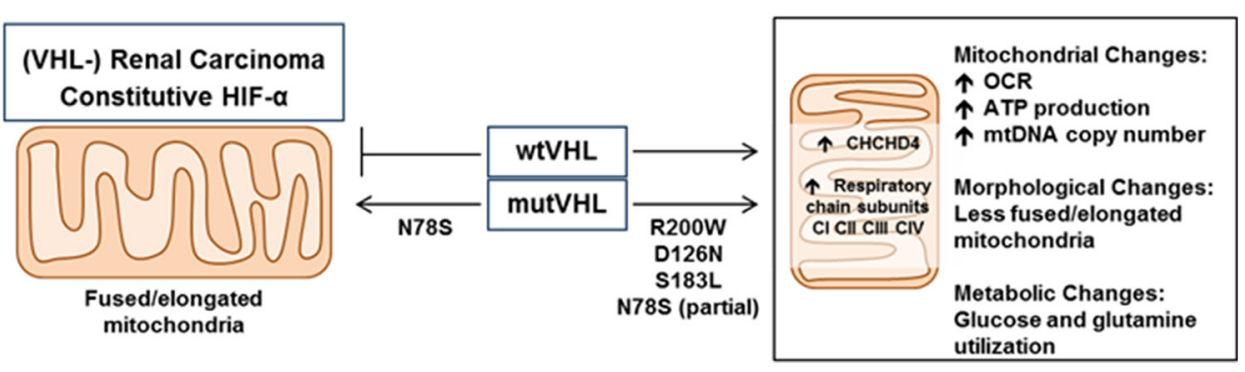

FIGURE 6 | pVHL mutants differentially regulate mitochondrial morphology and the mitochondrial network. (A) Confocal microscope images of RCC10 cells empty vector (pCMV), wild type $\mathrm{pVHL}(\mathrm{VHL})$, or pVHL mutants (R200W or N78S). Cells were fixed and mitochondria were stained for ATP5B (red). Nuclei were stained with DAPI (blue) and images were acquired with a Zeiss 510 META confocal laser scanning microscope using a $63 \times$ oil objective. Right panels show mitochondria (red) in 
FIGURE 6 | higher magnified images. (B-E) Graphs show analysis of mitochondria from ATP5B stained cells described in (A) using MiNA (39). Graphs show number of mitochondria that are unbranched (B) and branched (C), mean number of branched mitochondria per network (D) and the mitochondrial footprint normalized to pCMV control (E) for each cell line as indicated. $n=28$ ( $\mathrm{pCMV}$, VHL), $n=13$ (R200W), $n=14$ (N78S). Data are presented as mean \pm S.E.M. and analyzed using a one-way ANOVA with Tukey's post-hoc comparison. (n.s. $p>0.05,{ }^{* *} p<0.01$, and ${ }^{* \star *} p<0.001$ ). (F) pVHL-mediated regulation of CHCHD4 and mitochondrial function. pVHL re-expression in VHL-defective renal carcinoma cells leads to increased expression of $\mathrm{CHCHD} 4$ and respiratory chain subunits (from $\mathrm{Cl}$ to $\mathrm{CIV}$ ), increased respiration (OCR), ATP production, mtDNA copy number, changes in metabolism (glucose and glutamine), and changes in mitochondrial morphology (less fused/elongated). pVHL mutants exhibit differential effects.

containing mitochondria was removed and this step was repeated and supernatant again removed. Mitochondria were sedimented following centrifugation at $9,000 \mathrm{~g}$ for $10 \mathrm{~min}$. The supernatant was removed and stored (cytosolic fraction). The crude mitochondrial pellet was washed in homogenization buffer and centrifuged at 9,000 $\mathrm{g}$ for $10 \mathrm{~min}$. The pellet was lysed in $1 \%$ NP40 lysis (mitochondrial fraction). All steps were performed on ice and centrifugation was performed at $4^{\circ} \mathrm{C}$.

\section{Measurement of Total Cellular ATP}

Cells were counted and re-suspended in complete media. To determine ATP concentration Cell-Titer Glo reagent (Promega) was used as outlined in the manufacturer's instructions. Luminescence was quantified using a Tropix TR717 microplate reader.

\section{High-Resolution Respirometry}

Cellular oxygen consumption rates were measured using the Oxygraph 2K (O2K; Oroboros Instruments). Cells were trypsinised and re-suspended in HEPES-buffered DMEM and counted. Cells were added to each chamber and incubated at $37^{\circ} \mathrm{C}$ under constant stirring $(750 \mathrm{rpm})$. Cells were allowed to reach routine baseline respiration before the addition of the first compound. Two $\mu \mathrm{g} / \mathrm{ml}$ oligomycin, $0.5 \mu \mathrm{M}$ increments FCCP, $0.5 \mu \mathrm{M}$ rotenone, and $2.5 \mu \mathrm{M}$ antimycin $\mathrm{A}$ were injected in sequence and the rates of oxygen consumption assessed.

\section{Determination of mtDNA Copy Number}

Total DNA was isolated from cell pellets using QIAamp DNA blood mini kit (Qiagen). Abundance of mitochondrial DNA relative to nuclear genomic DNA was determined by qRT-PCR using primers for ND1 (mitochondrial, forward $5^{\prime}$-TCCTCTCC CGCTCTGCACCC- $3^{\prime}$ and reverse $5^{\prime}$-GGCGGGCCACCAAGG AGAAC-3') and beta-2-microglobulin ( $\beta 2 M$; nuclear, forward $5^{\prime}$ GCCCCAACGTTGTAGGCCCC- $3^{\prime}$ and reverse $5^{\prime}$ - AGCTAAG GTCGGGGCGGTGA-3'). Estimation of the number of copies of the mitochondrial genome relative to nuclear genome was based on the threshold cycle (Ct) as described previously (52).

\section{Immunostaining}

Cells were plated on glass coverslips ( $\varnothing 13 \mathrm{~mm}$, VWR) and incubated overnight. Cells were washed twice with PBS and fixed using 4\% paraformaldehyde in PBS for $15 \mathrm{~min}$. Cells were then washed twice with PBS and permeabilized for 10 min using $0.5 \%$ Triton X in PBS before being blocked with IFF (1\% BSA, 1\% FCS in PBS) for $1 \mathrm{~h}$. Coverslips were incubated overnight with primary antibody at $4^{\circ} \mathrm{C}$ before being washed and incubated with fluorophore conjugated secondary antibody for $1 \mathrm{~h}$. Coverslips were again washed twice with PBS and incubated in $1 \mu \mathrm{g} / \mathrm{ml}$
DAPI solution (Biotium) for $10 \mathrm{~min}$, washed and mounted using Fluoromount G (Southern Biotech). DAPI was excited at $405 \mathrm{~nm}$ and emitted fluorescence (peak $465 \mathrm{~nm}$ ) collected using 435485 band pass filter and red fluorescence captured following excitation at $543 \mathrm{~nm}$ and collected using a $560 \mathrm{~nm}$ long pass filter. Images were acquired with a Zeiss 510 META confocal laser scanning microscope using a $63 \times$ oil immersion objective.

\section{Confocal Live-Cell Imaging}

Cells were seeded on to glass coverslips (ø $22 \mathrm{~mm}, \mathrm{VWR}$ ) and Mitoview green (Biotium) used for mitochondrial imaging at a concentration of $100 \mathrm{nM}$. Mitoview green was excited at $488 \mathrm{~nm}$ and emitted fluorescence captured using a 505-530 band pass filter. Images were acquired with a Zeiss 510 META confocal laser scanning microscope using a $63 \times$ oil immersion objective.

\section{Analysis of Mitochondrial Network Morphology}

Fluorescently-labeled mitochondrial networks within cultured cells were analyzed using the Mitochondrial Network Analysis (MiNA) macro tool (39) in ImageJ. Pre-processing to enhance image quality was uniformly performed across images using the built-in contrast limited adaptive equalization, median filtering, and top-hat filtering options. Regions of interest containing single cells were selected for analysis and a mitochondrial footprint for each cell per image was generated. The mitochondrial footprint is the total area occupied by mitochondrial signal (i.e., mitochondrial structures) separated from the background, and is calculated by multiplying the number of pixels containing signal in the binarized image (only mitochondrial signal included) by the area of a pixel.

\section{Electron Microscopy}

Cells were seeded on to glass coverslips (ø $13 \mathrm{~mm}$, VWR), and fixed with $2 \%$ paraformaldehyde and $1.5 \%$ glutaraldehyde in $0.1 \mathrm{M}$ cacodylate buffer. Samples were post fixed with $1 \%$ osmium tetraoxide and $1.5 \%$ potassium ferrocyanine in $0.1 \mathrm{M}$ cacodylate buffer. Samples were dehydrated in a graded ethanol-water series, cleared in propylene oxide and infiltrated with agar-100 resin. Ultra-thin sections were cut and collected on 300-mesh grids and stained with lead citrate. Samples were viewed in a Joel 1010 transition electron microscope and images were acquired using a Gatan Orius CDD camera. Mitochondrial length was analyzed using ImageJ measure feature, calibrated using the scale bar of each image. Analysis of up to 15-20 mitochondria from 10 cells per condition was performed. Statistical significance was calculated using an unpaired two-tailed $t$-test. 


\section{Metabolomics Analyses to Determine the Levels of Intracellular Glucose and Glutamine and Their Uptake From the Culture Media}

For the intracellular glucose and glutamine measurements, cells were methanol fixed, and harvested by scraping, then phase separated by the serial addition of chloroform and water, followed by centrifugation. Divalent ions were removed by the addition then removal by centrifugation of Chelex 100. Cell extracts were prepared for ${ }^{1} \mathrm{H}$-MRS by freeze drying, followed by resuspension in $\mathrm{D}_{2} \mathrm{O}$ and $0.75 \%$ TSP (in $\mathrm{D}_{2} \mathrm{O}$ ) at a 14:1 ratio. For the glucose and glutamine uptake measurements, $1 \mathrm{H}$-MRS were performed on $500 \mu \mathrm{l}$ of culture media samples (starting media that has not been incubated with the cells and media samples that has been collected just before cell harvest for extractions) with the addition of $50 \mu \mathrm{l}$ of D2O and $50 \mu \mathrm{l}$ of $0.75 \%$ TSP. ${ }^{1} \mathrm{H}-\mathrm{MR}$ spectra of the extracted and media samples were acquired at $25^{\circ} \mathrm{C}$ using a pulse-acquired MR sequence with water suppression (1D NOESY presat sequence) in a broad-band-inverse NMR probe on a Bruker $500 \mathrm{MHz}$ NMR system.

\section{Sulforhodamine B (SRB) Assay}

Cells were plated in appropriate tissue culture vessels, and allowed to adhere overnight prior to treatment. At the end of incubation, media was removed and cells were fixed with $10 \%$ trichloroacetic acid (TCA) for $30 \mathrm{~min}$. TCA was washed with water, wells were allowed to air dry, and then an excess of $0.4 \%$ (w/v) SRB in $1 \%$ acetic acid was used to stain fixed cells for $>10$ min. Excess SRB was washed off with $1 \%$ acetic acid solution. Bound SRB was resuspended in a suitable volume of $10 \mathrm{mM}$ Tris, and absorbance of solution measured at $570 \mathrm{~nm}$. For proliferation assays, cells were plated on "day -1" in triplicate in 12 well plates, and cultured in maintenance DMEM overnight, after which "day 0" plates fixed with TCA. For galactose conditions, glucose media was replaced with glucose-free DMEM, supplemented with $4.5 \mathrm{~g} / \mathrm{L}$ galactose, $10 \% \mathrm{FCS}$, penicillin, streptomycin and Lglutamine, and $1 \mathrm{mM}$ pyruvate. Cells were then incubated for desired time points in either normoxia or hypoxia, followed by SRB assay.

\section{Experimental Design, Data Analysis, and Statistical Procedures}

Data are presented as mean \pm standard deviation (S.D) or standard error (S.E.M). Statistical analysis was performed using an unpaired two-tailed $t$-test in GraphPad Prism version 7.02

\section{REFERENCES}

1. Connor TM, Hoer S, Mallett A, Gale DP, Gomez-Duran A, Posse V, et al. Mutations in mitochondrial DNA causing tubulointerstitial kidney disease. PLoS Genet. (2017) 13:e1006620. doi: 10.1371/journal.pgen.1006620

2. Boland ML, Chourasia AH, Macleod KF. Mitochondrial dysfunction in cancer. Front Oncol. (2013) 3:292. doi: 10.3389/fonc.2013.00292

3. Murley A, Nunnari J. The emerging network of mitochondria-organelle contacts. Mol Cell (2016) 61:648-53. doi: 10.1016/j.molcel.2016.01.031 for Windows (La Jolla, California, USA) or otherwise indicated in figure legends. Statistical significance was determined using $P<0.05$.

\section{AUTHOR CONTRIBUTIONS}

TB and JMS designed and performed experiments, analyzed data, and contributed to writing the manuscript. LWT, CE, and SES performed experiments and analyzed data. Y-LC performed metabolomics analysis and analyzed data. MT performed electron microscopy. LAM performed MiNA analysis. BG performed bioinformatics analysis. PHM provided RCC10 pVHL mutant cells. GS and SV designed experiments. MA provided the concept for the study, designed experiments, analyzed data, wrote the manuscript, and acquired funding. All authors reviewed the manuscript.

\section{FUNDING}

TB was funded by a British Heart Foundation (BHF) (FS/09/051) and METOXIA FP7 (HEALTH-F2-2009-222741) awards to MA. JMS was funded by a Medical Research Council (MRC) Doctoral Training and Sackler award (RG70550) to MA. LWT was funded by MRC grants (MR/K002201/1, MR/K002201/2) to MA. CE was funded by a Cancer Research UK (CRUK) award (C7358/A19442) to MA. Y-LC was supported by the CR-UK and EPSRC Cancer Imaging Centre in association with the MRC and Department of Health (England) grant C1060/A10334, and CRUK grant C1060/A16464. LAM was funded by a CRUK Ph.D. Studentship (RG91141) award to MA. BG was funded by a CRUK Clinical Research Training Fellowship (RG85993) award to MA. SV and SES were funded by MRC grant: Medical Research Council (MC_UU_12022/7).

\section{ACKNOWLEDGMENTS}

Thanks to all members of the Ashcroft laboratory, including Rachel Morgan for technical support. We also thank Celeste Simon (University of Pennsylvania) for helpful discussions.

\section{SUPPLEMENTARY MATERIAL}

The Supplementary Material for this article can be found online at: https://www.frontiersin.org/articles/10.3389/fonc. 2018.00388/full\#supplementary-material

4. Friedman JR, Nunnari J. Mitochondrial form and function. Nature (2014) 505:335-43. doi: 10.1038/nature12985

5. Samanta D, Semenza GL. Maintenance of redox homeostasis by hypoxia-inducible factors. Redox Biol. (2017) 13:331-5. doi: 10.1016/j.redox.2017.05.022

6. Kaelin WG Jr, Ratcliffe PJ. Oxygen sensing by metazoans: the central role of the HIF hydroxylase pathway. Mol Cell (2008) 30:393-402. doi: 10.1016/j.molcel.2008. 04.009 
7. Semenza GL, Oxygen sensing, hypoxia-inducible factors, and disease pathophysiology. Annu Rev Pathol. (2014) 9:47-71. doi: 10.1146/annurev-pathol-012513-104720

8. Epstein AC, Gleadle JM, McNeill LA, Hewitson KS, O'Rourke J, Mole DR, et al. C. elegans EGL-9 and mammalian homologs define a family of dioxygenases that regulate HIF by prolyl hydroxylation. Cell (2001) 107:43-54. doi: 10.1016/S0092-8674(01)00507-4

9. Masson N, Willam C, Maxwell PH, Pugh CW, Ratcliffe PJ. Independent function of two destruction domains in hypoxia-inducible factor-alpha chains activated by prolyl hydroxylation. EMBO J. (2001) 20:5197-206. doi: 10.1093/emboj/20.18.5197

10. Maxwell PH, Wiesener MS, Chang GW, Clifford SC, Vaux EC, Cockman ME, et al. The tumour suppressor protein VHL targets hypoxia-inducible factors for oxygen-dependent proteolysis. Nature (1999) 399:271-5. doi: $10.1038 / 20459$

11. Jaakkola P, Mole DR, Tian YM, Wilson MI, Gielbert J, Gaskell SJ, et al. Targeting of HIF-alpha to the von Hippel-Lindau ubiquitylation complex by O2-regulated prolyl hydroxylation. Science (2001) 292:468-72. doi: 10.1126/science.1059796

12. Ivan M, Kondo K, Yang H, Kim W, Valiando J, Ohh M, Salic $\mathrm{A}$, et al. HIFalpha targeted for VHL-mediated destruction by proline hydroxylation: implications for O2 sensing. Science (2001) 292:464-8. doi: 10.1126/science. 1059817

13. Kaelin WG Jr. The VHL tumor suppressor gene: insights into oxygen sensing and cancer. Trans Am Clin Climatol Assoc. (2017) 128:298-307.

14. Shen C, Kaelin WG Jr. The VHL/HIF axis in clear cell renal carcinoma. Semin Cancer Biol. (2013) 23:18-25. doi: 10.1016/j.semcancer.2012.06.001

15. Iliopoulos O, Kibel A, Gray S, Kaelin WG Jr. Tumour suppression by the human von Hippel-Lindau gene product. Nat Med. (1995) 1:822-6. doi: $10.1038 / \mathrm{nm} 0895-822$

16. Raval RR, Lau KW, Tran MG, Sowter HM, Mandriota SJ, Li JL, et al. Contrasting properties of Hypoxia-Inducible Factor 1 (HIF-1) and HIF-2 in von hippel-lindau-associated renal cell carcinoma. Mol Cell Biol. (2005) 25:5675-86. doi: 10.1128/MCB.25.13.5675-5686.2005

17. Carroll VA, Ashcroft M. Role of hypoxia-inducible factor (HIF)-1alpha versus HIF-2alpha in the regulation of HIF target genes in response to hypoxia, insulin-like growth factor-I, or loss of von Hippel-Lindau function: implications for targeting the HIF pathway. Cancer Res. (2006) 66:6264-70. doi: 10.1158/0008-5472.CAN-05-2519

18. Hervouet E, Demont J, Pecina P, Vojtiskova A, Houstek J, Simonnet H, et al. A new role for the von Hippel-Lindau tumor suppressor protein: stimulation of mitochondrial oxidative phosphorylation complex biogenesis. Carcinogenesis (2005) 26:531-9. doi: 10.1093/carcin/bgi001

19. Craven RA, Hanrahan S, Totty N, Harnden P, Stanley AJ, Maher ER, et al. Proteomic identification of a role for the von Hippel Lindau tumour suppressor in changes in the expression of mitochondrial proteins and septin 2 in renal cell carcinoma. Proteomics (2006) 6:3880-93. doi: 10.1002/pmic.200500811

20. Zhang H, Gao P, Fukuda R, Kumar G, Krishnamachary B, Zeller KI, et al. HIF1 inhibits mitochondrial biogenesis and cellular respiration in VHL-deficient renal cell carcinoma by repression of C-MYC activity. Cancer Cell (2007) 11:407-20. doi: 10.1016/j.ccr.2007.04.001

21. Hervouet E, Cizkova A, Demont J, Vojtiskova A, Pecina P, Franssen-van Hal NL, et al. HIF and reactive oxygen species regulate oxidative phosphorylation in cancer. Carcinogenesis (2008) 29:1528-37. doi: 10.1093/carcin/ bgn 125

22. Yang J, Staples O, Thomas LW, Briston T, Robson M, Poon E, et al. Human CHCHD4 mitochondrial proteins regulate cellular oxygen consumption rate and metabolism and provide a critical role in hypoxia signaling and tumor progression. J Clin Invest. (2012) 122:600-11. doi: 10.1172/JCI 58780

23. Thomas LW, Staples O, Turmaine M, Ashcroft M. CHCHD4 regulates intracellular oxygenation and perinuclear distribution of mitochondria. Front Oncol. (2017) 7:71. doi: 10.3389/fonc.2017.00071

24. Hofmann S, Rothbauer U, Muhlenbein N, Baiker K, Hell K, Bauer MF. Functional and mutational characterization of human MIA40 acting during import into the mitochondrial intermembrane space. J Mol Biol. (2005) 353:517-28. doi: 10.1016/j.jmb.2005.08.064
25. Bien M, Longen S, Wagener N, Chwalla I, Herrmann JM, Riemer J. Mitochondrial disulfide bond formation is driven by intersubunit electron transfer in Erv1 and proofread by glutathione. Mol Cell (2010) 37:516-28. doi: $10.1016 /$ j.molcel.2010.01.017

26. Fischer M, Riemer J. The mitochondrial disulfide relay system: roles in oxidative protein folding and beyond. Int J Cell Biol. (2013) 2013:742923. doi: 10.1155/2013/742923

27. Erdogan AJ, Ali M, Habich M, Salscheider SL, Schu L, Petrungaro C, et al. The mitochondrial oxidoreductase $\mathrm{CHCHD} 4$ is present in a semioxidized state in vivo. Redox Biol. (2018) 17:200-6. doi: 10.1016/j.redox.2018. 03.014

28. Petrungaro C, Zimmermann KM, Kuttner V, Fischer M, Dengjel J, Bogeski I, Riemer J. The $\mathrm{Ca}(2+)$-dependent release of the Mia40-induced MICU1MICU2 dimer from MCU regulates mitochondrial $\mathrm{Ca}(2+)$ uptake. Cell Metab. (2015) 22:721-33. doi: 10.1016/j.cmet.2015.08.019

29. Backes S, Herrmann JM. Protein translocation into the intermembrane space and matrix of mitochondria: mechanisms and driving forces. Front Mol Biosci. (2017) 4:83. doi: 10.3389/fmolb.2017.00083

30. Friederich MW, Erdogan AJ, Coughlin CR II, Elos MT, Jiang H, O'Rourke CP, et al. Mutations in the accessory subunit NDUFB10 result in isolated complex I deficiency and illustrate the critical role of intermembrane space import for complex I holoenzyme assembly. Hum Mol Genet. (2017) 26:702-16. doi: 10.1093/hmg/ddw431

31. Bond J, Gale DP, Connor T, Adams S, de Boer J, Gascoyne DM, et al. Dysregulation of the HIF pathway due to VHL mutation causing severe erythrocytosis and pulmonary arterial hypertension. Blood (2011) 117:3699701. doi: 10.1182/blood-2010-12-327569

32. Gnarra JR, Tory K, Weng Y, Schmidt L, Wei MH, Li H, et al. Mutations of the VHL tumour suppressor gene in renal carcinoma. Nat Genet. (1994) 7:85-90. doi: 10.1038/ng0594-85

33. Cuperlovic-Culf M, Cormier K, Touaibia M, Reyjal J, Robichaud S, Belbraouet $\mathrm{M}$, et al. (1)H NMR metabolomics analysis of renal cell carcinoma cells: effect of VHL inactivation on metabolism. Int J Cancer (2016) 138:2439-49. doi: $10.1002 / \mathrm{ijc} .29947$

34. Crabtree HG. The carbohydrate metabolism of certain pathological overgrowths. Biochem J. (1928) 22:1289-98. doi: 10.1042/bj0221289

35. Krieg $M$, Haas $R$, Brauch $H$, Acker $T$, Flamme I, Plate KH. Upregulation of hypoxia-inducible factors HIF-1alpha and HIF-2alpha under normoxic conditions in renal carcinoma cells by von Hippel-Lindau tumor suppressor gene loss of function. Oncogene (2000) 19:5435-43. doi: 10.1038/sj.onc.1203938

36. Clifford SC, Cockman ME, Smallwood AC, Mole DR, Woodward ER, Maxwell PH, et al. Contrasting effects on HIF-1alpha regulation by disease-causing pVHL mutations correlate with patterns of tumourigenesis in von Hippel-Lindau disease. Hum Mol Genet. (2001) 10:1029-38. doi: $10.1093 / \mathrm{hmg} / 10.10 .1029$

37. Hickey MM, Lam JC, Bezman NA, Rathmell WK, Simon MC. von HippelLindau mutation in mice recapitulates Chuvash polycythemia via hypoxiainducible factor-2alpha signaling and splenic erythropoiesis. J Clin Invest. (2007) 117:3879-89. doi: 10.1172/JCI32614

38. Couve S, Ladroue C, Laine E, Mahtouk K, Guegan J, Gad S, et al. Genetic evidence of a precisely tuned dysregulation in the hypoxia signaling pathway during oncogenesis. Cancer Res. (2014) 74:6554-64. doi: 10.1158/0008-5472.CAN-14-1161

39. Valente AJ, Maddalena LA, Robb EL, Moradi F, Stuart JA. A simple ImageJ macro tool for analyzing mitochondrial network morphology in mammalian cell culture. Acta Histochem. (2017) 119:315-26. doi: 10.1016/j.acthis.2017.03.001

40. Dirckx N, Tower RJ, Mercken EM, Vangoitsenhoven R, Moreau-Triby C, Breugelmans $\mathrm{T}$, et al. Vhl deletion in osteoblasts boosts cellular glycolysis and improves global glucose metabolism. J Clin Invest. (2018) 128:1087-105. doi: 10.1172/JCI97794

41. Li M, Kim WY. Two sides to every story: the HIF-dependent and HIF-independent functions of pVHL. J Cell Mol Med. (2011) 15:187-95. doi: $10.1111 /$ j.1582-4934.2010.01238.x

42. Wettersten HI, Aboud OA, Lara PN Jr. Weiss RH. Metabolic reprogramming in clear cell renal cell carcinoma. Nat Rev Nephrol. (2017) 13:410-9. doi: $10.1038 /$ nrneph.2017.59 
43. Semenza GL. HIF-1 mediates the Warburg effect in clear cell renal carcinoma. J Bioenerg Biomembr. (2007) 39:231-4. doi: 10.1007/s10863-007-9081-2

44. Chau NM, Rogers P, Aherne W, Carroll V, Collins I, McDonald E, et al. Identification of novel small molecule inhibitors of hypoxia-inducible factor-1 that differentially block hypoxia-inducible factor-1 activity and hypoxia-inducible factor-1alpha induction in response to hypoxic stress and growth factors. Cancer Res. (2005) 65:4918-28. doi: 10.1158/0008-5472.CAN04-4453

45. Chen W, Hill H, Christie A, Kim MS, Holloman E, Pavia-Jimenez A, et al. Targeting renal cell carcinoma with a HIF-2 antagonist. Nature (2016) 539:112-7. doi: 10.1038/nature19796

46. Cho H, Du X, Rizzi JP, Liberzon E, Chakraborty AA, Gao W, et al. Ontarget efficacy of a HIF-2alpha antagonist in preclinical kidney cancer models. Nature (2016) 539:107-11. doi: 10.1038/nature19795

47. Weinberg SE, Chandel NS. Targeting mitochondria metabolism for cancer therapy. Nat Chem Biol. (2015) 11:9-15. doi: 10.1038/nchembio.1712

48. Galluzzi L, Kepp O, Vander Heiden MG, Kroemer G. Metabolic targets for cancer therapy. Nat Rev Drug Discov. (2013) 12:829-46. doi: 10.1038/nrd4145

49. Carroll VA, Ashcroft M. Regulation of angiogenic factors by HDM2 in renal cell carcinoma. Cancer Res. (2008) 68:545-52. doi: 10.1158/0008-5472.CAN-06-4738
50. Fellmann C, Hoffmann T, Sridhar V, Hopfgartner B, Muhar M, Roth M, et al. An optimized microRNA backbone for effective single-copy RNAi. Cell Rep. (2013) 5:1704-13. doi: 10.1016/j.celrep.2013.11.020

51. Schmittgen TD, Livak KJ. Analyzing real-time PCR data by the comparative CT method. Nat Protoc. (2008) 3:1101-8. doi: 10.1038/nprot.2008.73

52. Venegas V, Halberg M. Measurement of mitochondrial DNA copy number. In: Wong PDL-JC, editor. Mitochondrial Disorders. Humana Press (2012). p. 327-35. doi: 10.1007/978-1-61779-504-6_22

Conflict of Interest Statement: The authors declare that the research was conducted in the absence of any commercial or financial relationships that could be construed as a potential conflict of interest.

Copyright (C) 2018 Briston, Stephen, Thomas, Esposito, Chung, Syafruddin, Turmaine, Maddalena, Greef, Szabadkai, Maxwell, Vanharanta and Ashcroft. This is an open-access article distributed under the terms of the Creative Commons Attribution License (CC BY). The use, distribution or reproduction in other forums is permitted, provided the original author(s) and the copyright owner(s) are credited and that the original publication in this journal is cited, in accordance with accepted academic practice. No use, distribution or reproduction is permitted which does not comply with these terms. 